TITLE:

\title{
Measurement of the intrinsic electron neutrino component in the T2K neutrino beam with the ND280 detector
}

\section{$\operatorname{AUTHOR}(\mathrm{S}):$}

Bronner, C.; Hiraki, T.; Hirota, S.; Huang, K.; Ichikawa, A.K.; leki, K.; Kikawa, T.; ... Suzuki, K.; Takahashi, S.; T2K Collaboration

\section{CITATION:}

Bronner, C.... [et al]. Measurement of the intrinsic electron neutrino component in the T2K neutrino beam with the ND280 detector. Physical Review D 2014, 89(9): 092003.

\section{ISSUE DATE:}

2014-05-01

URL:

http://hdl.handle.net/2433/250813

RIGHT:

(C) 2014 American Physical Society 
PHYSICAL REVIEW D 89, 092003 (2014)

\section{Measurement of the intrinsic electron neutrino component in the T2K neutrino beam with the ND280 detector}

K. Abe ${ }^{46}$ J. Adam,${ }^{32}$ H. Aihara, ${ }^{45,23}$ T. Akiri, ${ }^{9}$ C. Andreopoulos, ${ }^{44}$ S. Aoki,${ }^{24}$ A. Ariga, ${ }^{2}$ T. Ariga, ${ }^{2}$ S. Assylbekov, ${ }^{8}$ D. Autiero, ${ }^{29}$ M. Barbi, ${ }^{39}$ G. J. Barker,${ }^{54}$ G. Barr, ${ }^{35}$ M. Bass, ${ }^{8}$ M. Batkiewicz,${ }^{13}$ F. Bay, ${ }^{11}$ S. W. Bentham,${ }^{26}$ V. Berardi, ${ }^{18}$ B. E. Berger, ${ }^{8}$ S. Berkman, ${ }^{4}$ I. Bertram, ${ }^{26}$ S. Bhadra,${ }^{58}$ F. d. M. Blaszczyk, ${ }^{28}$ A. Blondel,${ }^{12}$ C. Bojechko, ${ }^{51}$ S. Bordoni,${ }^{15}$ S. B. Boyd,${ }^{54}$ D. Brailsford, ${ }^{17}$ A. Bravar, ${ }^{12}$ C. Bronner, ${ }^{25}$ N. Buchanan, ${ }^{8}$ R. G. Calland,${ }^{27}$ J. Caravaca Rodríguez, ${ }^{15}$ S. L. Cartwright, ${ }^{42}$ R. Castillo, ${ }^{15}$ M. G. Catanesi, ${ }^{18}$ A. Cervera, ${ }^{16}$ D. Cherdack, ${ }^{8}$ G. Christodoulou, ${ }^{27}$ A. Clifton, ${ }^{8}$ J. Coleman, ${ }^{27}$ S. J. Coleman, ${ }^{7}$ G. Collazuol, ${ }^{20}$ K. Connolly, ${ }^{55}$ L. Cremonesi, ${ }^{38}$ A. Dabrowska, ${ }^{13}$ I. Danko, ${ }^{37}$ R. Das, ${ }^{8}$ S. Davis, ${ }^{55}$ P. de Perio, ${ }^{49}$ G. De Rosa, ${ }^{19}$ T. Dealtry, ${ }^{44,35}$ S. R. Dennis, ${ }^{54,44}$ C. Densham, ${ }^{44}$ F. Di Lodovico, ${ }^{38}$ S. Di Luise, ${ }^{11}$ O. Drapier, ${ }^{10}$ T. Duboyski ${ }^{38}$ K. Duffy, ${ }^{35}$ F. Dufour, ${ }^{12}$ J. Dumarchez ${ }^{36}$ S. Dytman, ${ }^{37}$ M. Dziewiecki, ${ }^{53}$ S. Emery, ${ }^{6}$ A. Ereditato, ${ }^{2}$ L. Escudero, ${ }^{16}$ A. J. Finch, ${ }^{26}$ L. Floetotto, ${ }^{41}$ M. Friend, ${ }^{14, \uparrow}$ Y. Fujii, ${ }^{14, \dagger}$ Y. Fukuda ${ }^{30}$ A. P. Furmanski, ${ }^{54}$ V. Galymov, ${ }^{6}$ S. Giffin, ${ }^{39}$ C. Giganti, ${ }^{36}$ K. Gilje, ${ }^{32}$ D. Goeldi, ${ }^{2}$ T. Golan, ${ }^{57}$ J. J. Gomez-Cadenas, ${ }^{16}$ M. Gonin, ${ }^{10}$ N. Grant,${ }^{26}$ D. Gudin, ${ }^{22}$ D. R. Hadley, ${ }^{54}$ A. Haesler, ${ }^{12}$ M. D. Haigh, ${ }^{54}$ P. Hamilton, ${ }^{17}$ D. Hansen, ${ }^{37}$ T. Hara ${ }^{24}$ M. Hartz, ${ }^{23,50}$

T. Hasegawa, ${ }^{14, \dagger}$ N. C. Hastings, ${ }^{39}$ Y. Hayato, ${ }^{46}$ C. Hearty, ${ }^{4,}$ R. L. Helmer, ${ }^{50}$ M. Hierholzer, ${ }^{2}$ J. Hignight, ${ }^{32}$ A. Hillairet, ${ }^{51}$ A. Himmel, ${ }^{9}$ T. Hiraki, ${ }^{25}$ S. Hirota, ${ }^{25}$ J. Holeczek, ${ }^{43}$ S. Horikawa, ${ }^{11}$ K. Huang, ${ }^{25}$ A. K. Ichikawa, ${ }^{25}$ K. Ieki, ${ }^{25}$ M. Ieva, ${ }^{15}$ M. Ikeda, ${ }^{46}$ J. Imber, ${ }^{32}$ J. Insler, ${ }^{28}$ T. J. Irvine, ${ }^{47}$ T. Ishida, ${ }^{14, \dagger}$ T. Ishii ${ }^{14, \dagger}$ S. J. Ives, ${ }^{17}$ E. Iwai, ${ }^{14}$ K. Iyogi,${ }^{46}$ A. Izmaylov, ${ }^{16,22}$ A. Jacob, ${ }^{35}$ B. Jamieson, ${ }^{56}$ R. A. Johnson, ${ }^{7}$ J. H. Jo, ${ }^{32}$ P. Jonsson, ${ }^{17}$ C. K. Jung, ${ }^{32,}{ }^{8}$ M. Kabirnezhad ${ }^{31}$ A. C. Kaboth, ${ }^{17}$ T. Kajita, ${ }^{47,8}$ H. Kakuno, ${ }^{48}$ J. Kameda, ${ }^{46}$ Y. Kanazawa, ${ }^{45}$ D. Karlen,${ }^{51,50}$ I. Karpikov ${ }^{22}$ E. Kearns,${ }^{3,23, \$}$ M. Khabibullin ${ }^{22}$

A. Khotjantsev, ${ }^{22}$ D. Kielczewska, ${ }^{52}$ T. Kikawa, ${ }^{25}$ A. Kilinski, ${ }^{31}$ J. Kim, ${ }^{4}$ J. Kisiel, ${ }^{43}$ P. Kitching, ${ }^{1}$ T. Kobayashi, ${ }^{14, \uparrow}$ L. Koch, ${ }^{41}$ A. Kolaceke, ${ }^{39}$ A. Konaka, ${ }^{50}$ L. L. Kormos, ${ }^{26}$ A. Korzenev, ${ }^{12}$ K. Koseki, ${ }^{14, \dagger}$ Y. Koshio ${ }^{33,8}$ I. Kreslo, ${ }^{2}$ W. Kropp ${ }_{5}^{5}$ H. Kubo, ${ }^{25}$ Y. Kudenko, ${ }^{22, \|}$ S. Kumaratunga, ${ }^{50}$ R. Kurjata, ${ }^{53}$ T. Kutter, ${ }^{28}$ J. Lagoda, ${ }^{31}$ K. Laihem, ${ }^{41}$ I. Lamont,${ }^{26}$ E. Larkin,${ }^{54}$ M. Laveder, ${ }^{20}$ M. Lawe, ${ }^{42}$ M. Lazos, ${ }^{27}$ K. P. Lee, ${ }^{47}$ T. Lindner, ${ }^{50}$ C. Lister, ${ }^{54}$ R. P. Litchfield ${ }^{54}$ A. Longhin,${ }^{20}$ L. Ludovici, ${ }^{21}$ M. Macaire, ${ }^{6}$ L. Magaletti, ${ }^{18}$ K. Mahn, ${ }^{50}$ M. Malek, ${ }^{17}$ S. Manly, ${ }^{40}$ A. D. Marino, ${ }^{7}$ J. Marteau, ${ }^{29}$ J. F. Martin, ${ }^{49}$

T. Maruyama, ${ }^{14, \dagger}$ J. Marzec, ${ }^{53}$ E. L. Mathie, ${ }^{39}$ V. Matveev, ${ }^{22}$ K. Mavrokoridis,${ }^{27}$ E. Mazzucato, ${ }^{6}$ M. McCarthy, ${ }^{4}$

N. McCauley, ${ }^{27}$ K. S. McFarland, ${ }^{40}$ C. McGrew, ${ }^{32}$ C. Metelko, ${ }^{27}$ M. Mezzetto, ${ }^{20}$ P. Mijakowski, ${ }^{31}$ C. A. Miller, ${ }^{50}$ A. Minamino ${ }^{25}$ O. Mineev, ${ }^{22}$ S. Mine, ${ }^{5}$ A. Missert, ${ }^{7}$ M. Miura, ${ }^{46,8}$ L. Monfregola, ${ }^{16}$ S. Moriyama, ${ }^{46,8}$ Th. A. Mueller, ${ }^{10}$ A. Murakami, ${ }^{25}$ M. Murdoch, ${ }^{27}$ S. Murphy, ${ }^{11}$ J. Myslik ${ }^{51}$ T. Nagasaki, ${ }^{25}$ T. Nakadaira, ${ }^{14, \dagger}$ M. Nakahata ${ }^{46,23}$ T. Nakai, ${ }^{34}$ K. Nakamura, ${ }^{23,14, \uparrow}$ S. Nakayama, ${ }^{46,8}$ T. Nakaya, ${ }^{25,23}$ K. Nakayoshi, ${ }^{14, \dagger}$ D. Naples, ${ }^{37}$ C. Nielsen, ${ }^{4}$ M. Nirkko, ${ }^{2}$ K. Nishikawa, ${ }^{14, \dagger}$ Y. Nishimura, ${ }^{47}$ H. M. O’Keeffe, ${ }^{26}$ R. Ohta, ${ }^{14,7}$ K. Okumura, ${ }^{47,23}{ }^{37}$. Okusawa, ${ }^{34}$ W. Oryszczak, ${ }^{52}$ S. M. Oser ${ }^{4}$ R. A. Owen ${ }^{38}$ Y. Oyama,${ }^{14, \dagger}$ V. Palladino, ${ }^{19}$ J. Palomino, ${ }^{32}$ V. Paolone, ${ }^{37}$ D. Payne,${ }^{27}$ O. Perevozchikov ${ }^{28}$ J. D. Perkin, ${ }^{42}$ Y. Petrov, ${ }^{4}$ L. Pickard ${ }^{42}$ E. S. Pinzon Guerra ${ }^{58}$ C. Pistillo, ${ }^{2}$ P. Plonski, ${ }^{53}$ E. Poplawska, ${ }^{38}$ B. Popov, ${ }^{36,9}$ M. Posiadala, ${ }^{52}$ J.-M. Poutissou, ${ }^{50}$ R. Poutissou, ${ }^{50}$ P. Przewlocki, ${ }^{31}$ B. Quilain, ${ }^{10}$ E. Radicioni, ${ }^{18}$ P. N. Ratoff ${ }^{26}$ M. Ravonel, ${ }^{12}$ M. A. M. Rayner, ${ }^{12}$ A. Redij, ${ }^{2}$ M. Reeves, ${ }^{26}$ E. Reinherz-Aronis, ${ }^{8}$ F. Retiere, ${ }^{50}$ A. Robert, ${ }^{36}$ P. A. Rodrigues ${ }^{40}$ P. Rojas, ${ }^{8}$ E. Rondio, ${ }^{31}$ S. Roth,${ }^{41}$ A. Rubbia, ${ }^{11}$ D. Ruterbories, ${ }^{40}$ R. Sacco, ${ }^{38}$ K. Sakashita, ${ }^{14, \dagger}$ F. Sánchez, ${ }^{15}$ F. Sato, ${ }^{14}$ E. Scantamburlo, ${ }^{12}$ K. Scholberg, ${ }^{9,8}$ S. Schoppmann, ${ }^{41}$ J. Schwehr, ${ }^{8}$ M. Scott, ${ }^{50}$ Y. Seiya, ${ }^{34}$ T. Sekiguchi ${ }^{14, \dagger}$ H. Sekiya, ${ }^{46,}$ D. Sgalaberna, ${ }^{11}$ M. Shiozawa, ${ }^{46,23}$ S. Short, ${ }^{17}$ Y. Shustrov, ${ }^{22}$ P. Sinclair, ${ }^{17}$ B. Smith, ${ }^{17}$ R. J. Smith, ${ }^{35}$ M. Smy, J. T. Sobczyk,${ }^{57}$ H. Sobel,,${ }^{5,23}$ M. Sorel, ${ }^{16}$ L. Southwell, ${ }^{26}$ P. Stamoulis, ${ }^{16}$ J. Steinmann, ${ }^{41}$ B. Still, ${ }^{38}$ Y. Suda, ${ }^{45}$ A. Suzuki, ${ }^{24}$ K. Suzuki, ${ }^{25}$ S. Y. Suzuki, ${ }^{14, \dagger}$ Y. Suzuki, ${ }^{46,23}$ T. Szeglowski, ${ }^{43}$ R. Tacik, ${ }^{39,50}$ M. Tada, ${ }^{14, \dagger}$ S. Takahashi, ${ }^{25}$ A. Takeda, ${ }^{46}$ Y. Takeuchi, ${ }^{24,23}$ H. K. Tanaka, ${ }^{46,8}$ H. A. Tanaka, ${ }^{4,}$ M. M. Tanaka,,${ }^{14, \dagger}$ D. Terhorst, ${ }^{41}$ R. Terri ${ }^{38}$ L. F. Thompson, ${ }^{42}$ A. Thorley, ${ }^{27}$ S. Tobayama, ${ }^{4}$ W. Toki ${ }^{8}$ T. Tomura ${ }^{46}{ }^{2}$ Y. Totsuka, ${ }^{*}$ C. Touramanis, ${ }^{27}$ T. Tsukamoto ${ }^{14,+}$ M. Tzanov, ${ }^{28}$ Y. Uchida, ${ }^{17}$ K. Ueno, ${ }^{46}$ A. Vacheret, ${ }^{35}$ M. Vagins, ${ }^{23,5}$ G. Vasseur, ${ }^{6}$ T. Wachala, ${ }^{13}$ A. V. Waldron, ${ }^{3,}$ C. W. Walter, ${ }^{9,8}$ D. Wark, ${ }^{44,17}$ M. O. Wascko, ${ }^{17}$ A. Weber, ${ }^{44,35}$ R. Wendell, ${ }^{46,8}$ R. J. Wilkes ${ }^{55}$ M. J. Wilking, ${ }^{50}$ C. Wilkinson, ${ }^{42}$ Z. Williamson, ${ }^{35}$ J. R. Wilson, ${ }^{38}$ R. J. Wilson, ${ }^{8}$ T. Wongjirad, ${ }^{9}$ Y. Yamada, ${ }^{14, \dagger}$ K. Yamamoto, ${ }^{34}$ C. Yanagisawa, ${ }^{32, * *}$ S. Yen, ${ }^{50}$ N. Yershov, ${ }^{22}$ M. Yokoyama, ${ }^{45,8}$ T. Yuan, ${ }^{7}$ M. Yu, ${ }^{58}$ A. Zalewska, ${ }^{13}$ J. Zalipska, ${ }^{31}$ L. Zambelli, ${ }^{36}$ K. Zaremba, ${ }^{53}$ M. Ziembicki, ${ }^{53}$ E. D. Zimmerman, ${ }^{7}$ M. Zito, ${ }^{6}$ and J. Żmuda ${ }^{57}$

(T2K Collaboration)

\footnotetext{
${ }^{1}$ University of Alberta, Centre for Particle Physics, Department of Physics, Edmonton, Alberta, Canada

${ }^{2}$ University of Bern, Albert Einstein Center for Fundamental Physics, Laboratory for High Energy Physics (LHEP), Bern, Switzerland

${ }^{3}$ Boston University, Department of Physics, Boston, Massachusetts, USA

${ }^{4}$ University of British Columbia, Department of Physics and Astronomy, Vancouver, British Columbia, Canada

${ }^{5}$ University of California, Irvine, Department of Physics and Astronomy, Irvine, California, USA
} 
${ }^{6}$ IRFU, CEA Saclay, Gif-sur-Yvette, France

${ }^{7}$ University of Colorado at Boulder, Department of Physics, Boulder, Colorado, USA

${ }^{8}$ Colorado State University, Department of Physics, Fort Collins, Colorado, USA

${ }^{9}$ Duke University, Department of Physics, Durham, North Carolina, USA

${ }^{10}$ Ecole Polytechnique, IN2P3-CNRS, Laboratoire Leprince-Ringuet, Palaiseau, France

${ }^{11}$ ETH Zurich, Institute for Particle Physics, Zurich, Switzerland

${ }^{12}$ University of Geneva, Section de Physique, DPNC, Geneva, Switzerland

${ }^{13}$ H. Niewodniczanski Institute of Nuclear Physics PAN, Cracow, Poland

${ }^{14}$ High Energy Accelerator Research Organization (KEK), Tsukuba, Ibaraki, Japan

${ }^{15}$ Institut de Fisica d'Altes Energies (IFAE), Bellaterra (Barcelona), Spain

${ }^{16}$ IFIC (CSIC \& University of Valencia), Valencia, Spain

${ }^{17}$ Imperial College London, Department of Physics, London, United Kingdom

${ }^{18}$ INFN Sezione di Bari and Università e Politecnico di Bari,

Dipartimento Interuniversitario di Fisica, Bari, Italy

${ }^{19}$ INFN Sezione di Napoli and Università di Napoli, Dipartimento di Fisica, Napoli, Italy

${ }^{20}$ INFN Sezione di Padova and Università di Padova, Dipartimento di Fisica, Padova, Italy

${ }^{21}$ INFN Sezione di Roma and Università di Roma "La Sapienza," Roma, Italy

${ }^{22}$ Institute for Nuclear Research of the Russian Academy of Sciences, Moscow, Russia

${ }^{23}$ University of Tokyo, Kavli Institute for the Physics and Mathematics of the Universe (WPI),

Todai Institutes for Advanced Study, Kashiwa, Chiba, Japan

${ }^{24}$ Kobe University, Kobe, Japan

${ }^{25}$ Kyoto University, Department of Physics, Kyoto, Japan

${ }^{26}$ Lancaster University, Physics Department, Lancaster, United Kingdom

${ }^{27}$ University of Liverpool, Department of Physics, Liverpool, United Kingdom

${ }^{28}$ Louisiana State University, Department of Physics and Astronomy, Baton Rouge, Louisiana, USA

${ }^{29}$ Université de Lyon, Université Claude Bernard Lyon 1, IPN Lyon (IN2P3), Villeurbanne, France

${ }^{30}$ Miyagi University of Education, Department of Physics, Sendai, Japan

${ }^{31}$ National Centre for Nuclear Research, Warsaw, Poland

${ }^{32}$ State University of New York at Stony Brook, Department of Physics and Astronomy, Stony Brook, New York, USA

${ }^{33}$ Okayama University, Department of Physics, Okayama, Japan

${ }^{34}$ Osaka City University, Department of Physics, Osaka, Japan

${ }^{35}$ Oxford University, Department of Physics, Oxford, United Kingdom

${ }^{36} U P M C$, Université Paris Diderot, CNRS/IN2P3,

Laboratoire de Physique Nucléaire et de Hautes Energies (LPNHE), Paris, France

${ }^{37}$ University of Pittsburgh, Department of Physics and Astronomy, Pittsburgh, Pennsylvania, USA

${ }^{38}$ Queen Mary University of London, School of Physics and Astronomy, London, United Kingdom

${ }^{39}$ University of Regina, Department of Physics, Regina, Saskatchewan, Canada

${ }^{40}$ University of Rochester, Department of Physics and Astronomy, Rochester, New York, USA

${ }^{41}$ RWTH Aachen University, III. Physikalisches Institut, Aachen, Germany

${ }^{42}$ University of Sheffield, Department of Physics and Astronomy, Sheffield, United Kingdom

${ }^{43}$ University of Silesia, Institute of Physics, Katowice, Poland

${ }^{44}$ STFC, Rutherford Appleton Laboratory, Harwell Oxford, and Daresbury Laboratory,

Warrington, United Kingdom

${ }^{45}$ University of Tokyo, Department of Physics, Tokyo, Japan

${ }^{46}$ University of Tokyo, Institute for Cosmic Ray Research, Kamioka Observatory, Kamioka, Japan

${ }^{47}$ University of Tokyo, Institute for Cosmic Ray Research, Research Center for Cosmic Neutrinos, Kashiwa, Japan

${ }^{48}$ Tokyo Metropolitan University, Department of Physics, Tokyo, Japan

${ }^{49}$ University of Toronto, Department of Physics, Toronto, Ontario, Canada ${ }^{50}$ TRIUMF, Vancouver, British Columbia, Canada

${ }^{51}$ University of Victoria, Department of Physics and Astronomy, Victoria, British Columbia, Canada

${ }^{52}$ University of Warsaw, Faculty of Physics, Warsaw, Poland

${ }^{53}$ Warsaw University of Technology, Institute of Radioelectronics, Warsaw, Poland

${ }^{54}$ University of Warwick, Department of Physics, Coventry, United Kingdom

${ }^{55}$ University of Washington, Department of Physics, Seattle, Washington, USA

${ }^{56}$ University of Winnipeg, Department of Physics, Winnipeg, Manitoba, Canada

${ }^{57}$ Wroclaw University, Faculty of Physics and Astronomy, Wroclaw, Poland

${ }^{58}$ York University, Department of Physics and Astronomy, Toronto, Ontario, Canada

(Received 11 March 2014; published 5 May 2014; corrected 7 May 2014) 
The T2K experiment has reported the first observation of the appearance of electron neutrinos in a muon neutrino beam. The main and irreducible background to the appearance signal comes from the presence in the neutrino beam of a small intrinsic component of electron neutrinos originating from muon and kaon decays. In T2K, this component is expected to represent $1.2 \%$ of the total neutrino flux. A measurement of this component using the near detector (ND280), located $280 \mathrm{~m}$ from the target, is presented. The charged current interactions of electron neutrinos are selected by combining the particle identification capabilities of both the time projection chambers and electromagnetic calorimeters of ND280. The measured ratio between the observed electron neutrino beam component and the prediction is $1.01 \pm 0.10$ providing a direct confirmation of the neutrino fluxes and neutrino cross section modeling used for T2K neutrino oscillation analyses. Electron neutrinos coming from muons and kaons decay are also separately measured, resulting in a ratio with respect to the prediction of $0.68 \pm 0.30$ and $1.10 \pm 0.14$, respectively.

DOI: 10.1103/PhysRevD.89.092003

PACS numbers: 14.60.Pq, 13.15.+g

\section{INTRODUCTION}

The T2K (Tokai-to-Kamioka) experiment [1] is a long baseline neutrino oscillation experiment that uses an intense muon neutrino beam produced at the Japan Proton Accelerator Research Complex (J-PARC) in Tokai. The primary goals of the experiment are the precise determination of the oscillation parameter $\theta_{13}$ via electron neutrino appearance, and of the parameters $\theta_{23}$ and $\Delta m_{32}^{2}$ via muon neutrino disappearance. Neutrino interactions are observed at a near detector, ND280, where the flavor composition of the incoming neutrino flux is not expected to be affected by oscillation, and at the far detector, Super-Kamiokande (SK), where oscillation significantly affects the composition.

The T2K baseline, the neutrino beam configuration, and the ability of the far detector to distinguish electrons from muons results in excellent sensitivity for $\nu_{e}$ appearance. The $\nu_{\mu} \rightarrow \nu_{e}$ oscillation probability depends on $\theta_{13}$, and on subleading effects that depend on the $\delta_{\mathrm{CP}}$ phase and on the mass hierarchy [2]. Recently $\mathrm{T} 2 \mathrm{~K}$ reported the first observation of electron neutrino appearance with a $7.3 \sigma$ significance, by observing 28 electron neutrino events compared to a background expectation of $4.92 \pm 0.55$ events for $\theta_{13}=0$ [3]. Among those background events, 3.2 are expected to be due to the intrinsic $\nu_{e}$ beam component, an irreducible background to the electron neutrino appearance search.

The intrinsic $\nu_{e}$ in the beam are an unavoidable product of conventional neutrino beams where pions and kaons, produced by the interaction of a proton beam with a target, decay to form a $\nu_{\mu}$ beam. When the muons and kaons decay, a small component of $\nu_{e}$ is produced in addition to the $\nu_{\mu}$. In the T2K case $\nu_{e}$ are expected to represent about $1.2 \%$ of the total neutrino flux [4]. This component will be the main source of background for all the proposed longbaseline neutrino oscillation experiments [5-7] aiming to measure $C P$ violation in the leptonic sector by precisely measuring $\nu_{e}\left(\bar{\nu}_{e}\right)$ appearance in a $\nu_{\mu}\left(\bar{\nu}_{\mu}\right)$ beam. A direct measurement of this component performed at ND280 is presented in this paper.

ND280 is a magnetized detector located at a distance of $280 \mathrm{~m}$ from the target. For this analysis neutrino charged current (CC) interactions in the fine grained detectors (FGDs) [8] are selected. The combination of the particle identification (PID) capabilities of three time projection chambers (TPC) [9] and a set of electromagnetic calorimeters (ECals) [10] is used to distinguish electrons from muons, allowing the selection of a clean sample of $\nu_{e} \mathrm{CC}$ interactions with a purity of about $65 \%$. The background is dominated by photon conversions producing $e^{+} e^{-}$pairs in the FGD.

In the $\mathrm{T} 2 \mathrm{~K}$ oscillation analyses the measurement of the spectra of $\nu_{\mu} \mathrm{CC}$ interactions at ND280 is used to constrain the uncertainties on the unoscillated neutrino fluxes and on the neutrino cross section parameters. The $\nu_{\mu} \mathrm{CC}$ sample constrains also the $\nu_{e}$ flux and cross section because of the significant correlations between the $\nu_{\mu}$ and the $\nu_{e}$ fluxes, which originate from the same hadrons. The $\nu_{\mu}$ and $\nu_{e}$ cross sections are expected to be the same, except for radiative corrections and the different lepton mass [11].

The beam $\nu_{e}$ component is directly measured at ND280 and it is compared with the expectations when fluxes and cross section uncertainties are constrained by the same fit to the ND280 $\nu_{\mu}$ CC sample used for the oscillation analyses.

\footnotetext{
Deceased.

Also at J-PARC, Tokai, Japan.

Also at Institute of Particle Physics, Canada.

${ }^{\S}$ Also at Kavli IPMU (WPI), the University of Tokyo, Japan.

"Also at Moscow Institute of Physics and Technology and National Research Nuclear University "MEPhI," Moscow, Russia.

"Also at JINR, Dubna, Russia.

Also at BMCC/CUNY, Science Department, New York, New York, USA.
} 
This measurement directly confirms the validity of the procedure used in all $\mathrm{T} 2 \mathrm{~K}$ oscillation analyses.

The paper is organized as follows. In Sec. II the T2K experiment, the flux prediction and the neutrino interaction cross section model are described, and in Sec. III ND280 and the detectors used in this analysis are introduced. The electron neutrino selection is then described in Sec. IV and the control of the backgrounds entering the analysis is shown in Sec. V. A description of the systematics is given in Sec. VI and the fit used to extract the beam $\nu_{e}$ component is shown in Sec. VII. Finally the results are given in Sec. VIII and a summary in Sec. IX.

\section{EXPERIMENTAL OVERVIEW}

$\mathrm{T} 2 \mathrm{~K}$ is the first long-baseline experiment designed to observe electron neutrino appearance in a nearly pure muon neutrino beam. The neutrino beam is produced by the J-PARC accelerator complex where protons are accelerated up to $31 \mathrm{GeV} / \mathrm{c}$ before being extracted in $5 \mu \mathrm{s}$ long spills with a repetition rate that has been decreased from 3.6 to $2.6 \mathrm{~s}$ over the data-taking periods. The spill consists of 8 bunches ( 6 during the first data-taking period), each $15 \mathrm{~ns}$ wide. The protons strike a $91.4 \mathrm{~cm}$ long graphite target, producing hadrons, mainly pions and kaons. The positively charged particles are focused by a series of three magnetic horns operating at $250 \mathrm{kA}$ before entering a $96 \mathrm{~m}$ long decay volume where they decay producing mainly muon neutrinos. A small fraction of the kaons, and the muons produced by pion decay, can also decay producing electron neutrinos. Most of the surviving charged particles are stopped in a beam dump at the end of the decay volume. A muon monitor (MUMON) situated downstream of the beam dump measures the profile of high energy muons not stopped by the beam dump, monitoring the stability of the beam intensity and the direction of the beam. The neutrinos are sampled $280 \mathrm{~m}$ and $295 \mathrm{~km}$ from the target, at the ND280 near detector and SK far detector, respectively.

The direction of the proton beam and the axis of the target and horns is $2.5^{\circ}$ away from the direction to SK, giving a narrow band $\nu_{\mu}$ beam peaked at $0.6 \mathrm{GeV}$ towards SK. This corresponds to the oscillation maximum for the $295 \mathrm{~km}$ baseline. T2K is the first experiment designed to use this configuration, called the off-axis technique [12]. This configuration also has the advantage of reducing the beam $\nu_{e}$ component in the oscillation region and the high energy neutrino flux which contributes to backgrounds in the oscillation analyses.

The near detector complex is comprised of an on-axis detector (INGRID) [13] and an off-axis detector (ND280) that will be described in detail in the next section. SK is a $50 \mathrm{kt}$ cylindrical water Cherenkov detector. The water tank is optically separated into two concentric detectors, an inner detector and an outer detector, both instrumented with photomultipliers. Charged particles emitted from neutrino interactions produce photons through the Cherenkov effect and ring-shaped patterns are detected on the walls by the photomultipliers. The ring pattern is used to identify the type of the particle. Until recently, the main backgrounds to the electron neutrino appearance searches came from the intrinsic beam $\nu_{e}$ and from neutral current (NC) interactions in which a $\pi^{0}$ in the final state $\left(\mathrm{NC} \pi^{0}\right)$ is produced but only one electronlike ring is reconstructed [14,15]. A new reconstruction algorithm [3] has been developed to substantially reduce the $\mathrm{NC} \pi^{0}$ background, leaving the intrinsic beam $\nu_{e}$ component as the main background to the $\nu_{e}$ appearance analysis.

In this paper a direct measurement of the $\nu_{e}$ beam component is presented. All the data collected between January 2010 and May 2013 are used for the analysis. The data are subdivided into different run periods as shown in Table I. A small fraction of run III data $(\sim 15 \%)$ was collected with magnetic horns operating at $205 \mathrm{kA}$ instead of the nominal $250 \mathrm{kA}$, while for run I data only one subset of the ECal, the downstream module, was installed and operated. The remaining modules were installed and commissioned before the start of run II. The simulated data used in this analysis corresponds to more than ten times the p.o.t. of the data, and the various experimental conditions of the different data-taking periods are reproduced.

\section{A. Flux prediction}

A good knowledge of the initial neutrino fluxes at ND280 and at SK is fundamental for all the physics analyses in T2K. The $\nu_{\mu}\left(\nu_{e}\right)$ components of the beam are mainly produced through charged pion (muon) and kaon decays.

In the $\mathrm{T} 2 \mathrm{~K}$ simulation the interaction of the primary proton beam and the propagation of secondary particles in the carbon target are simulated with FLUKA [16]. The flux prediction is based on the hadron production measurements performed by NA61/SHINE, a fixed target experiment at the CERN SPS in which a proton beam of the same energy as the T2K beam interacts with a thin carbon target $(2 \mathrm{~cm}$ long) or with a T2K replica target $(91.4 \mathrm{~cm}$ long) [17]. The charged hadrons produced in the proton-carbon interactions are tracked by a system of TPCs, and their production cross sections as functions of momentum and angle are measured. The NA61/SHINE measurements cover most of the

TABLE I. Definition of T2K runs and the number of protons on target (p.o.t.) used in the analysis.

\begin{tabular}{lcc}
\hline \hline T2K run & Dates & ND280 \\
\hline Run I & Jan. 2010 $\left(\times 10^{19}\right)$ \\
Run II & Nov. 2010-Mar. 2010 2011 & 1.7 \\
Run III & Mar. 2012-Jun. 2012 & 7.9 \\
Run IV & Jan. 2013-May 2013 & 15.6 \\
Total & Jan. 2010-May 2013 & 33.8 \\
\hline \hline
\end{tabular}


relevant hadron production phase space for the T2K flux. The observed production cross section of pions [18] and kaons [19] on the thin target are used in the T2K flux simulation to reduce the uncertainties on the flux prediction [4]. Measurements from other experiments (Eichten et al. [20] and Allaby et al. [21]) are used to reduce the uncertainty of the particle production in the region not covered by NA61/SHINE.

The propagation of particles through the elements of the beam line is simulated with GEANT3 [22]. The particles are propagated through the horns' magnetic field and may interact with the surrounding materials. Particle decays into neutrinos are simulated as well as the interactions in the decay volume and the beam dump. The modeling of hadronic interactions is done using the GCALOR model [23]. The beam direction, its intensity and the beam profile are measured by the INGRID and MUMON detectors. The neutrino fluxes are described by a covariance matrix in bins of neutrino energy and type. The uncertainty on the $\nu_{\mu}$ flux is below $12 \%$ for neutrino energies around $0.6 \mathrm{GeV}$. The expected $\nu_{e}$ flux and its uncertainty at ND280 are shown in Figs. 1 and 2. Most of the intrinsic beam $\nu_{e}$ component comes from the decay of $\mu^{+}\left(\mu^{+} \rightarrow e^{+} \bar{\nu}_{\mu} \nu_{e}\right)$ produced by the pions' decay and from charged and neutral kaons. The charged kaons produce $\nu_{e}$ via the decays $K^{+} \rightarrow \pi^{0} e^{+} \nu_{e}$ that have a branching ratio of $5.1 \%$, while the neutral kaons produce $\nu_{e}$ through the decays $K_{L}^{0} \rightarrow \pi^{-} e^{+} \nu_{e}$ have a branching ratio of $40.5 \%$. The $\nu_{e}$ from muon decays contribute to most of the flux in the low energy region, below $1.5 \mathrm{GeV}$, while above that energy almost all of the $\nu_{e}$ flux comes from kaon decays. The $\nu_{e}$ from pion decays only contribute to about $1 \%$ of the total $\nu_{e}$ flux (Fig. 1). A more detailed discussion of the uncertainties contributing to the $\nu_{e}$ flux (Fig. 2) can be found in [4]. As the physics processes leading to $\nu_{e}$ from muon decay and from kaon decay are different, the analysis presented in this paper extracts a measurement of their separate contributions to the flux, as well as the inclusive flux of $\nu_{e}$.

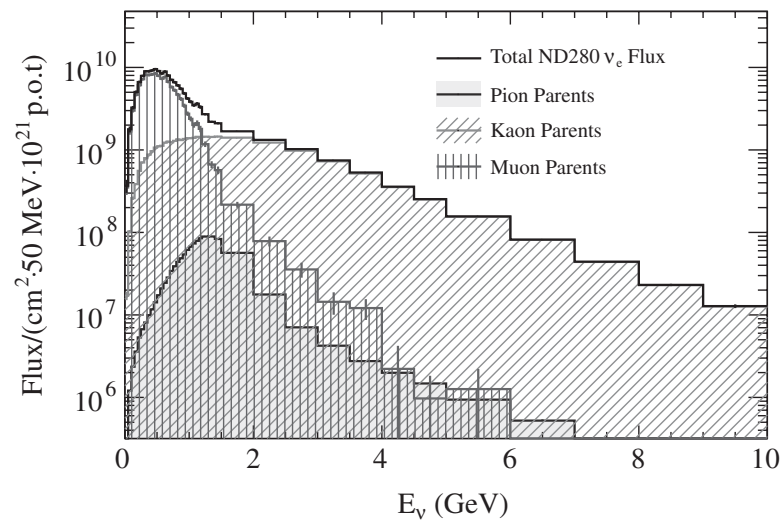

FIG. 1. The prediction of the $\nu_{e}$ flux at ND280 broken down by the neutrino parent particle type [4].

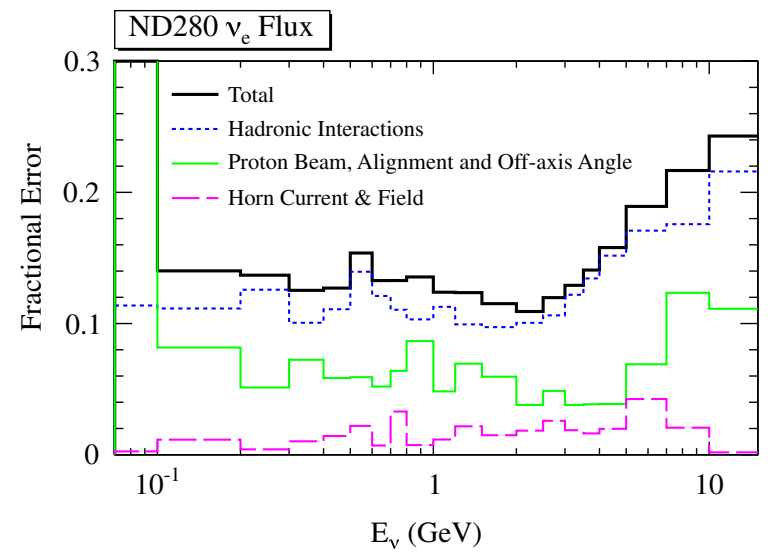

FIG. 2 (color online). The $\nu_{e}$ flux uncertainties at ND280 [4].

\section{B. Neutrino interaction model}

Neutrino interactions in ND280 are simulated using the NEUT [24] event generator. This generator covers the range of neutrino energies from several tens of $\mathrm{MeV}$ to hundreds of $\mathrm{TeV}$, and it simulates the full range of nuclear targets used in ND280. In the simulation, neutrino interactions are generated in the entire ND280 volume on both active and inactive targets, providing the necessary information for the signal and for the backgrounds coming from interactions occurring outside of the ND280 inner detectors. A complete description of the models used in the T2K simulation is given in [25].

The dominant cross section process at the peak of the T2K beam energy is charged current quasielastic scattering (CCQE): $\nu_{l}+N \rightarrow l+N$, while at higher energies, above the pion production threshold, single pion production $(\mathrm{CC} 1 \pi)$ and deep inelastic scattering (DIS) contribute to the total charged current cross section.

In NEUT, CCQE interactions are simulated using the model of Llewellyn Smith [26], with the nuclear effects described by the relativistic Fermi gas model of Smith and Moniz [27]. The form factors describing the vector and the axial masses are parametrized with $M_{V}=0.84 \mathrm{GeV}$ and $M_{A}=1.21 \mathrm{GeV}$. The Fermi momentum is set to $217(225) \mathrm{MeV} / \mathrm{c}$ and the binding energy to 25 (27) $\mathrm{MeV}$ for carbon (oxygen).

The pion production is simulated in NEUT using the model of Rein and Sehgal [28]. Below neutrino energies of $2 \mathrm{GeV}, 18$ resonances and their interference terms are simulated. For $20 \%$ of the $\Delta$ resonances NEUT simulates pionless decay in which the $\Delta$ deexcites without emitting pions.

Multipion and DIS processes are simulated using the GRV98 parton distribution functions [29]. If the invariant mass of the hadronic system $(W)$ is in the range $1.3<$ $W<2.0 \mathrm{GeV} / \mathrm{c}^{2}$ only pion multiplicities greater than one are considered to avoid double counting with the Rein and Sehgal model. For $W>2.0 \mathrm{GeV} / \mathrm{c}^{2}$ PYTHIA/JETSET [30] is used, applying the corrections in the small $Q^{2}$ 
region developed by Bodek and Yang [31]. Additional details on the NEUT simulation can be found in [1].

\section{Neutrino interaction uncertainties}

The modeling of the neutrino interactions constitutes an important source of systematic uncertainties for all T2K analyses. A detailed description of the uncertainties can be found in [15]. Only a brief summary of the systematic uncertainties is given here.

CCQE model uncertainty.-Recent measurements of CCQE scattering in the $1 \mathrm{GeV}$ region [32] show large discrepancies on the measurement of the axial mass $M_{A}^{\mathrm{QE}}$ with respect to older measurements. The strategy that is chosen in T2K analyses is to allow the ND280 $\nu_{\mu}$ CC samples to constrain this parameter, including a large prior uncertainty $\left(\sigma_{M_{A}^{\mathrm{OE}}}=0.43 \mathrm{GeV}\right)$ to account for the difference between the NEUT nominal value and the NEUT best fit to the MiniBooNE data. Additional degrees of freedom are allowed by three independent CCQE normalization factors $\left(x_{1,2,3}^{\mathrm{QE}}\right)$ for different neutrino energy ranges. Below $1.5 \mathrm{GeV}$ an uncertainty of $11 \%$ is assigned to $x_{1}^{\mathrm{QE}}$, corresponding to the uncertainty of the MiniBooNE flux. The other two normalization factors, $x_{2}^{\mathrm{QE}}$ for $1.5<E_{\nu}<$ $3.5 \mathrm{GeV}$ and $x_{3}^{\mathrm{QE}}$ for $E_{\nu}>3.5 \mathrm{GeV}$ are given a prior uncertainty of $30 \%$ to account for the discrepancy between MiniBooNE and NOMAD data [33].

Pion production.-For single pion production a joint fit to the MiniBooNE measurements of charged current single $\pi^{+}$production $\left(\mathrm{CC} 1 \pi^{+}\right)$[34], charged current single $\pi^{0}$ production $\left(\mathrm{CC} 1 \pi^{0}\right)$ [35], and neutral current single $\pi^{0}$ production $\left(\mathrm{NC} 1 \pi^{0}\right)$ [36] using NEUT has been performed, varying several parameters.

The parameters varied include the axial mass in the Rein and Sehgal model $M_{A}^{\mathrm{RES}}$, the normalization of $\mathrm{CC} 1 \pi\left(x_{1}^{\mathrm{CC} 1} \pi\right.$ for $E_{\nu}<2.5 \mathrm{GeV}$ and $x_{2}^{\mathrm{CC} 1 \pi}$ for $E_{\nu}>2.5 \mathrm{GeV}$ ), and the normalization of $\mathrm{NC} 1 \pi^{0}\left(x^{\mathrm{NC} 1 \pi^{0}}\right)$.

Contributions to the MiniBooNE samples from CC multipion, $\mathrm{NC}$ coherent interactions, $\mathrm{NC}$ charged pion interactions and $\mathrm{NC}$ multipion are relatively small and they are included in the analysis described here with a large prior uncertainty. For charged current coherent pion production a $100 \%$ normalization uncertainty $\left(x^{\mathrm{CC}} \mathrm{coh}\right)$, motivated by the nonobservation of the process in the few-GeV energy range by K2K [37] and SciBooNE [38], is assigned. For neutral current charged pion production and all other NC interactions, including DIS, a 30\% normalization uncertainty is introduced ( $\left.x^{\mathrm{NC} o t h}\right)$.

A $20 \%$ uncertainty on the fraction of $\Delta$ that deexcites without emitting pions $\left(x^{\pi \text {-less }}\right)$ is also included.

Finally for CC multipion/DIS interactions an energy dependent uncertainty is added ( $\left.x^{\mathrm{CC} \text { other}}\right)$, applying a weight $w$ with the form $w=1+x^{\mathrm{CC} \text { other }} / E_{\nu}(\mathrm{GeV})$. $x^{\mathrm{CC} \text { other }}$ is allowed to vary around a nominal value of 0 with a prior uncertainty of 0.4 .

Nuclear model uncertainties.-NEUT models nuclei with a relativistic Fermi gas model (RFG) using the Fermi momentum $p_{F}$ determined from electron scattering data. The uncertainty on $p_{F}$ is $30 \mathrm{MeV} / \mathrm{c}$, covering possible discrepancies in the CCQE cross section at low $Q^{2}$. The uncertainty is applied independently for interactions on carbon and oxygen targets.

Alternatives to the RFG model of the nuclei are considered by making comparisons to a spectral function nuclear model implemented in the NuWro neutrino interaction generator [39]. The discrepancy in CCQE interaction models using the RFG and spectral function are assigned as the uncertainty and represented by the parameter $x_{\mathrm{SF}}$, which linearly varies the predicted lepton kinematics between the $\operatorname{RFG}\left(x_{\mathrm{SF}}=0\right)$ and spectral function $\left(x_{\mathrm{SF}}=1\right)$ models.

Final state interactions (FSI) model tuning.-The NEUT FSI model includes parameters which alter the microscopic pion interaction probabilities in the nucleus. The central value of these parameters and their uncertainties are determined from fits to pion scattering data [40].

The cross section model parameters and their uncertainties are summarized in Table II. These uncertainties are used as prior uncertainties in the fit, along with the flux uncertainties to the ND280 fit to the $\nu_{\mu} \mathrm{CC}$ samples. As will be shown in Sec. VIC, the flux systematic uncertainties are

TABLE II. The parameters in the NEUT cross section model along with their nominal values and uncertainties prior to the analysis of ND280 data.

\begin{tabular}{lcc}
\hline \hline Parameter & Nominal value & Uncertainty \\
\hline$M_{A}^{\mathrm{QE}}(\mathrm{GeV})$ & 1.21 & 0.45 \\
$x_{1}^{\mathrm{QE}}$ & 1.00 & 0.11 \\
$x_{2}^{\mathrm{QE}}$ & 1.00 & 0.30 \\
$x_{3}^{\mathrm{QE}}$ & 1.00 & 0.30 \\
$x_{\mathrm{SF}}$ & 0.0 & 1.0 \\
$p_{F}\left({ }^{12} C\right)(\mathrm{MeV} / \mathrm{c})$ & 217 & 30 \\
$p_{F}\left({ }^{16} O\right)(\mathrm{MeV} / \mathrm{c})$ & 225 & 30 \\
$E_{B}\left({ }^{12} C\right)(\mathrm{MeV})$ & 25 & 3 \\
$E_{B}\left({ }^{16} O\right)(\mathrm{MeV})$ & 27 & 3 \\
$M_{A}^{\mathrm{RES}}(\mathrm{GeV})$ & 1.41 & 0.11 \\
$x_{1}^{\mathrm{CC} 1 \pi}$ & 1.15 & 0.32 \\
$x_{2}^{\mathrm{CC} 1 \pi}$ & 1.00 & 0.40 \\
$x^{\mathrm{NC} 1 \pi^{0}}$ & 0.96 & 0.32 \\
$x^{\pi-l e s s}$ & 0.20 & 0.20 \\
$x^{\mathrm{CC} \text { coh }}$ & 1.00 & 1.00 \\
$x^{\mathrm{NC} \text { other }}$ & 1.00 & 0.30 \\
$x^{\mathrm{CC} \text { other }}(\mathrm{GeV})$ & 0.00 & 0.40 \\
\hline \hline
\end{tabular}


reduced by the measurements of $\nu_{\mu} \mathrm{CC}$ interactions in ND280.

\section{THE ND280 DETECTOR}

The off-axis ND280 detector is a magnetized multipurpose detector located at the same off-axis angle as SK, at a distance of $280 \mathrm{~m}$ from the T2K target. The main purpose of ND280 is to measure the properties of $\nu_{\mu}$ and $\nu_{e} \mathrm{CC}$ interactions before oscillation, reducing uncertainties in the $\mathrm{T} 2 \mathrm{~K}$ oscillation analyses. It is also used to measure neutrino cross sections.

The layout of ND280 is shown in Fig. 3 and a complete description can be found in [1]. It is composed of a number of subdetectors installed inside the refurbished UA1/ NOMAD magnet that provides a magnetic field of $0.2 \mathrm{~T}$. The subdetectors are side muon range detectors [41] installed in the magnet yokes to track high angle muons, a $\pi^{0}$ detector (P0D) [42] explicitly built to measure neutrino interactions with the production of $\pi^{0}$ in the final state, and a tracking system. The tracking detector is composed of two FGDs used as the target for the neutrino interactions, and three TPCs. The tracker and the P0D are surrounded by a set of ECals. In this analysis the tracking detector, downstream ECal (DsECal) and the barrel ECal modules are used. The DsECal is installed downstream of the tracker system while the barrel ECal surrounds the tracker and consists of six different modules (two installed at the top of the tracker, two at the bottom and one at each side).

The first (upstream) FGD is composed of extruded polystyrene scintillator bars with layers oriented alternately in the $x$ and $y$ directions (defined in Fig. 3), allowing three dimensional tracking of the charged particles. The second FGD has the same structure, but the polystyrene bars are interleaved with water layers to allow for the measurement of neutrino interactions on water.

The TPCs consist of an inner box filled with Ar: $\mathrm{CF}_{4}: \mathrm{iC}_{4} \mathrm{H}_{10}$ and an outer box filled with $\mathrm{CO}_{2}$. Each

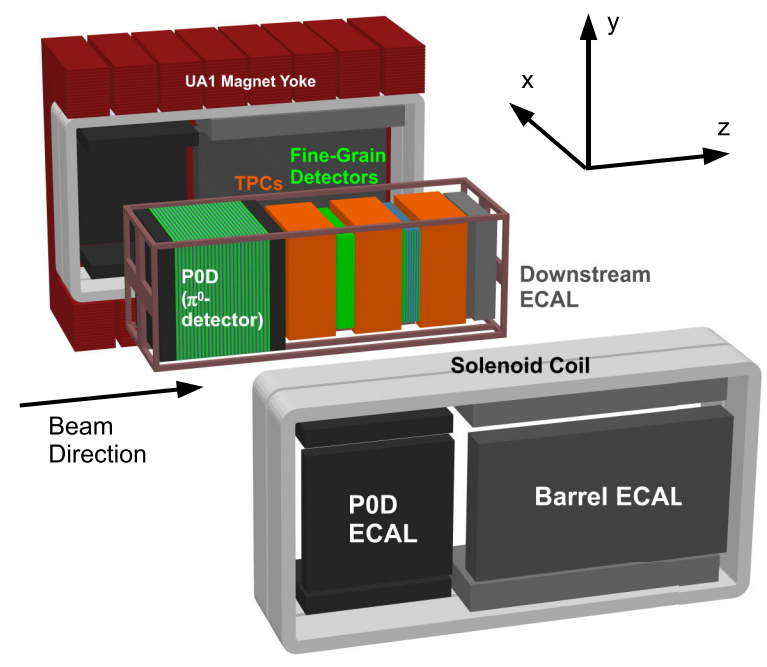

FIG. 3 (color online). A schematic view of the ND280 detector. side of the TPCs is instrumented with 12 MicroMEGAS modules arranged in two columns. Each MicroMEGAS is segmented into 1728 pads arranged in 48 rows and 36 columns, allowing a 3D reconstruction of charged particles produced in neutrino interactions.

The ECals are sampling calorimeters consisting of layers of $1 \mathrm{~cm}$ of plastic scintillator, divided into bars $4 \mathrm{~cm}$ wide, separated by $1 \mathrm{~mm}$ layers of lead. Alternating layers are aligned orthogonally to one another to provide three dimensional reconstruction of tracks and showers. The DsECal consists of 34 layers with readout from both ends of the scintillator bars. The barrel ECal has 31 layers with readout from both ends (one end) on the bars parallel (perpendicular) to the beam direction.

For the analysis described in this paper, neutrino interactions in both the FGDs are selected by requiring at least one track to enter the downstream TPC. A combination of TPC and ECal (when available) PID is used to select electrons, rejecting most of the muon background produced by the dominant $\nu_{\mu} \mathrm{CC}$ interactions in the FGD.

\section{A. TPC reconstruction and PID performance}

To reconstruct tracks in the TPC, the ionization signals on pads that exceed a threshold are saved as waveforms. Waveforms coincident in time and on adjacent pads in the vertical direction are joined to form clusters. Contiguous clusters are then combined to form track candidates and the kinematic parameters of the track are obtained with a maximum-likelihood fit to the observed charge distribution. After track reconstruction in the TPC, signals in the FGD are matched to the TPC tracks.

The PID in the TPC is based on the measurement of the ionization produced by charged particles crossing the gas. To perform particle identification in the TPC, the ionization in each cluster is corrected for the track length sampled by the pad column. Using $70 \%$ of the lowest charge deposits on the pads, a mean ionization value is calculated and is compared to that expected for particle type $i$ at the reconstructed momentum. This comparison is used to form the "pull" $\delta_{i}$ (the difference between the measured mean ionization and the expected one divided by the resolution). The resolution depends on the number of samples and path length with a typical resolution for muons of $8 \%$.

The deposited energy as a function of the reconstructed momentum for negatively charged particles starting in the FGD, compared with the expected curves from the simulation is shown in Fig. 4. In the energy region of interest for $\mathrm{T} 2 \mathrm{~K}$, the ionization difference between electrons and muons is $30 \%-40 \%$ allowing good separation between the two particles. Figure 5 shows the distribution of the pulls in two samples: the first is a sample of muons which cross the detector whose selection is described in Sec. VA, and the second is a sample of electrons and positrons selected as described in Sec. V B. 
K. $\mathrm{ABE}$ et al.

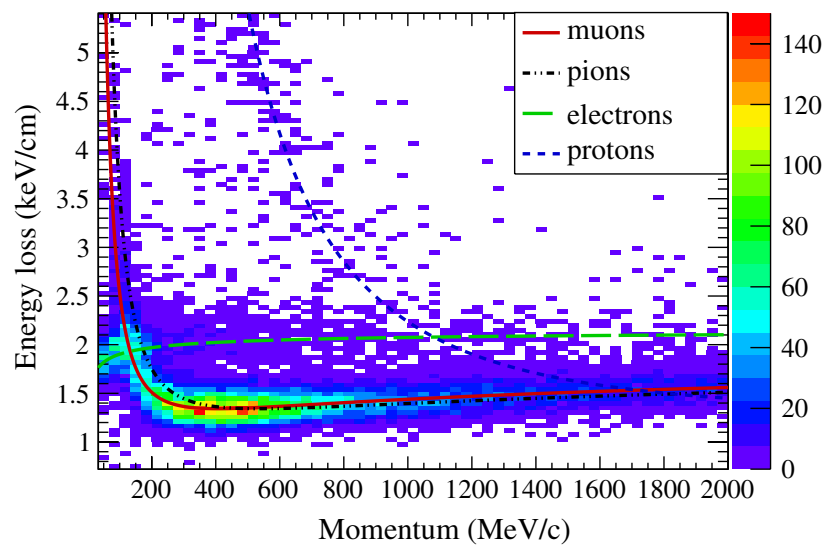

FIG. 4 (color online). TPC ionization energy loss per unit length as a function of the reconstructed momentum for negatively charged particles as measured by the TPCs (blocks) and the expected dependencies (curves).

\section{B. ECal reconstruction and PID performance}

Each ECal module has scintillator bars in two orientations. The reconstruction is performed by forming two sets of $2 \mathrm{D}$ clusters, one for each orientation, then combining them to form a $3 \mathrm{D}$ cluster. The $2 \mathrm{D}$ objects are built by clustering together adjacent hits. If more than one $2 \mathrm{D}$ object exists in a particular orientation, the choice of which should be used in the 3D object is based on a likelihood statistic combining time, position and charge information, aided by the extrapolation of in-time TPC tracks.

After an ECal cluster is reconstructed, PID statistics to classify the cluster are calculated. In particular, $R_{\mathrm{MIP} / \mathrm{EM}}$ is a statistic to separate electromagnetic showers and minimally ionizing tracks and is a likelihood ratio using characteristics that distinguish tracks and showers:

(i) circularity: clusters due to tracks are expected to be long and thin, while showers are expected to have a more spherical shape;

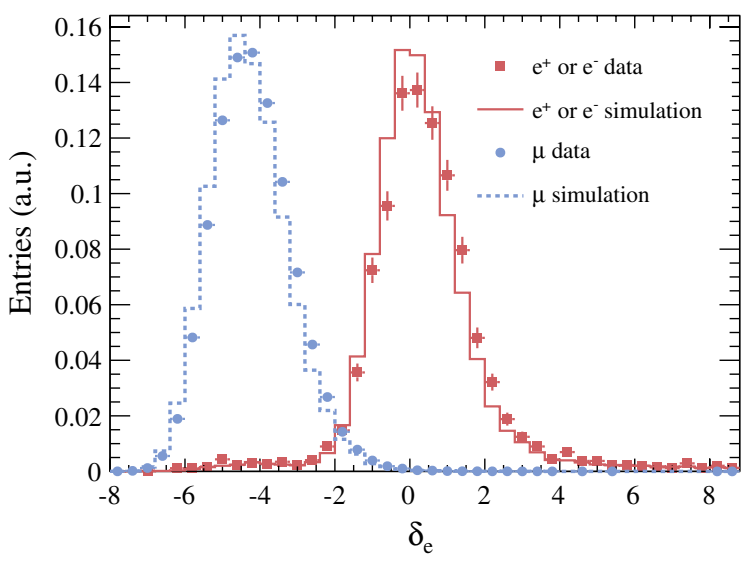

FIG. 5 (color online). TPC PID pulls in the electron hypothesis $\left(\delta_{e}\right)$ for electrons or positrons coming from photon conversions and for muons in data (points) and in the simulation (lines).
PHYSICAL REVIEW D 89, 092003 (2014)

(ii) charge distribution: electromagnetic showers have a highly nonuniform charge distribution compared to a minimally ionizing track. The charge distribution is parametrized using the ratio of the second and first moments and the ratio of the highest charge to the lowest charge layer;

(iii) charge ratio between first quarter and last quarter of the track: it is expected to be one for minimally ionizing tracks which deposit energy uniformly, greater than 1 for electromagnetic showers and less than 1 for highly ionizing particles such as protons which deposit most of their energy at the end of the track.

Samples of simulated electrons and muons are used to generate probability density functions (PDFs) that are used to construct the likelihood ratio. Figure 6 shows the $R_{\text {MIP/EM }}$ statistic in data and simulation for samples of $e^{+} e^{-}$from photon conversions and from crossing muons.

The energy deposited in the ECal $\left(E_{\mathrm{EM}}\right)$ is used for particles with reconstructed momenta in the TPC larger than $1 \mathrm{GeV} / \mathrm{c}$, to discriminate between electrons and muons. A charged particle that enters the ECal from the TPC has momentum measured in the tracker and this can be compared to the energy deposited in the ECal. Energy is reconstructed under the hypothesis that the energy deposit is due to an electromagnetic shower. A maximumlikelihood fit for the shower energy is constructed using the following variables:

(i) the total visible energy in the cluster: the total energy deposited into the scintillator is strongly correlated to the energy of the particle responsible for the EM shower and this parameter dominates the energy measurement in the ECal;

(ii) the RMS and the skewness of the deposited energy: these parameters provide additional information that refines the energy measurement.

The fit uses PDFs constructed from simulated photons at energies from $50 \mathrm{MeV}$ to $25 \mathrm{GeV}$, with the majority of

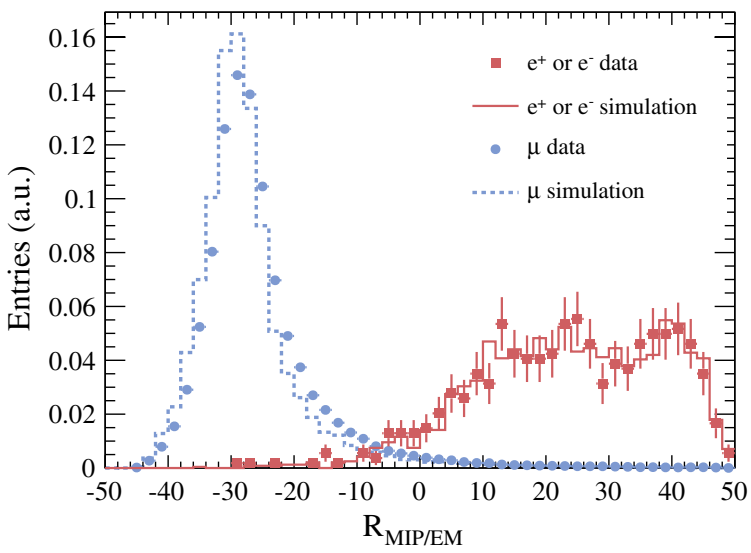

FIG. 6 (color online). Distribution of $R_{\mathrm{MIP} / \mathrm{EM}}$ for electrons or positrons coming from photon conversions, and for muons in the downstream ECal in data (points) and in the simulation (lines). 
photons below $2 \mathrm{GeV}$. The energy resolution for electrons at $1 \mathrm{GeV}$ is approximately $10 \%$.

\section{SELECTION OF ELECTRON NEUTRINO CC EVENTS AT ND280}

The signal events for this analysis are $\nu_{e} \mathrm{CC}$ interactions occurring in FGD1 or FGD2. Events in which there are electronlike tracks starting in either FGD are selected, and additional cuts are applied to reduce the contamination from photons converting into an $e^{+} e^{-}$pair in an FGD. The events are then split into separate CCQE-like and CCnonQE-like samples. A typical $\nu_{e} \mathrm{CC}$ candidate selected in the analysis is shown in Fig. 7.

After requiring a good beam spill and good ND280 data quality - all subdetectors were functioning correctly-the reconstructed objects in each spill are split into 8 time bunches ( 6 for run I). For each bunch the highest momentum negatively charged track is selected as the lepton candidate. If this track does not start in the fiducial volume (FV) of one of the FGDs the event is rejected. The FGD fiducial volume is defined by removing the outer $48 \mathrm{~mm}$ at each edge in $x$ and $y$ (distance equivalent to five scintillator bars) and the front $21 \mathrm{~mm}(7 \mathrm{~mm})$ at the begin of the FGD1 (FGD2), corresponding to the first $x-y(x)$ layer.

The track is also rejected if the reconstructed momentum is smaller than $200 \mathrm{MeV} / \mathrm{c}$ as that region is dominated by background from photon conversions. To ensure good TPC PID performance the selected track needs to have at least 36 reconstructed clusters in the TPC, corresponding to tracks crossing at least half of the TPC in the direction parallel to the beam.

Applying these criteria, $79 \%$ of the tracks are expected to be muons and just $6.5 \%$ electrons (see the inset in Fig. 8).

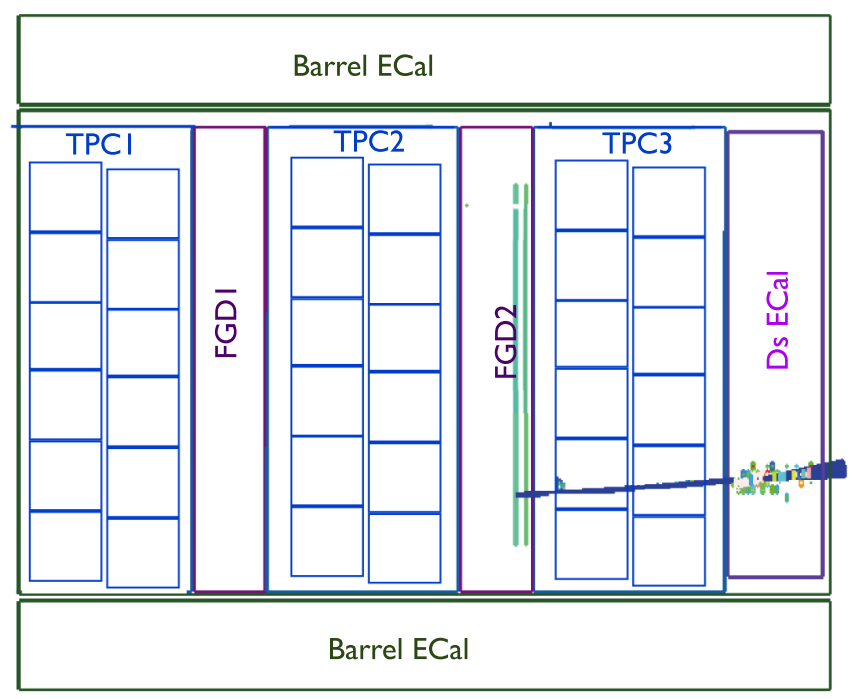

FIG. 7 (color online). Side view of a $\nu_{e}$ CCQE-like event in FGD2 with an electronlike track reconstructed in TPC3 and showering in the downstream ECal.
To select electrons, the TPC and ECal PID capabilities are combined. The PID criteria applied depend upon which subdetectors are used for the track reconstruction:

(i) if the electron candidate does not enter the ECal, the energy loss in the TPC is required to be electronlike $\left(-1<\delta_{e}<2\right)$, not muonlike $\left(\left|\delta_{\mu}\right|>2.5\right)$ and not pionlike $\left(\left|\delta_{\pi}\right|>2\right)$. This selection is also used for all tracks with reconstructed momentum in the TPC below $300 \mathrm{MeV} / \mathrm{c}$ as the ECal PID is not optimized for such low energy particles;

(ii) for tracks entering the ECal, the TPC PID is relaxed, only requiring an electronlike track $\left(-2<\delta_{e}<2.5\right)$. The ECal particle identification criteria depend on the momentum of the track as it enters the ECal module. For tracks with a momentum greater than $1 \mathrm{GeV} / \mathrm{c}$, the energy deposited in the ECal module is used to separate electromagnetic showers from minimum ionizing particles. Tracks are required to have $E_{\mathrm{EM}}>1100 \mathrm{MeV}$. For lower-momentum
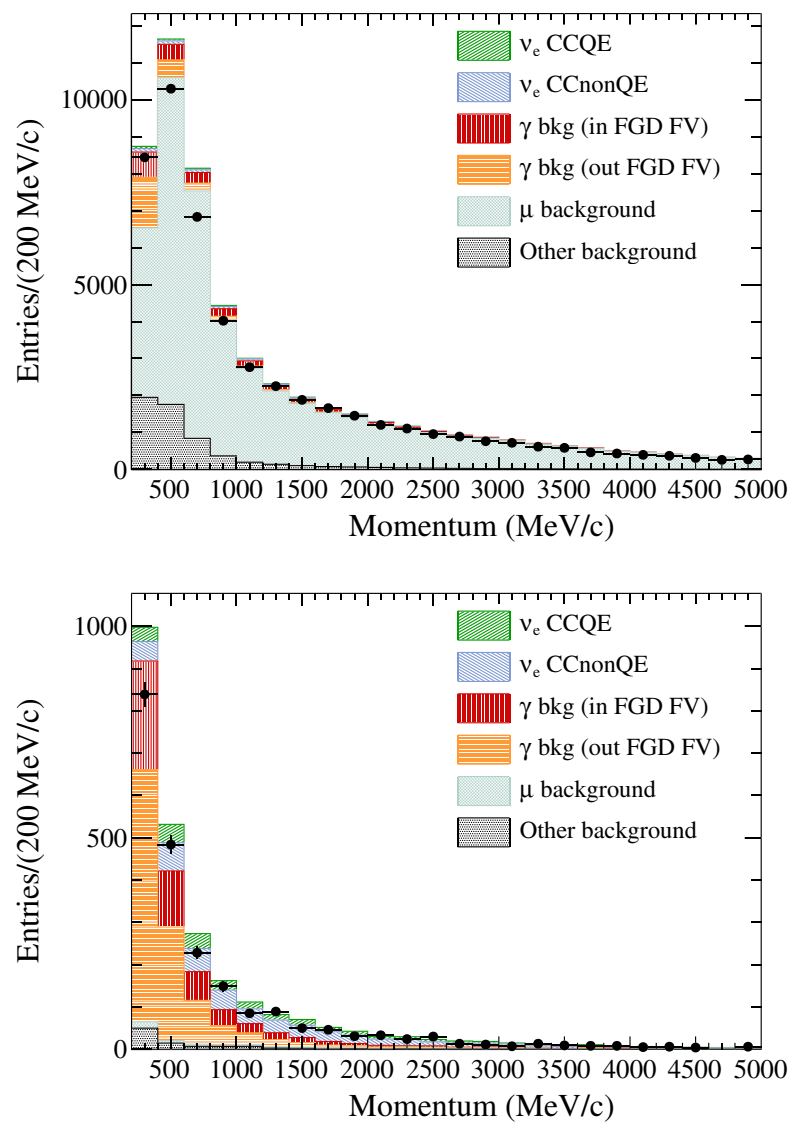

FIG. 8 (color online). Reconstructed electron momentum of events before (top) and after (bottom) the PID selection. The signal is divided into $\nu_{e}$ producing CCQE or CCnonQE interactions. The background is divided into photon conversions produced by neutrino interaction inside or outside the FGD, misidentified muon background and other background (mainly pions and protons). The error on the points is the statistical error on the data. 
K. $\mathrm{ABE}$ et al.

TABLE III. Fraction of electrons entering each PID branch, and efficiency and purity of the PID selection.

\begin{tabular}{|c|c|c|c|c|}
\hline \multirow[b]{2}{*}{ Category } & \multicolumn{2}{|c|}{ FGD1 vertices } & \multicolumn{2}{|c|}{ FGD2 vertices } \\
\hline & Events & Efficiency (\%) & Events & Efficiency $(\%)$ \\
\hline & $(\%)$ & [purity (\%)] & $(\%)$ & [purity (\%)] \\
\hline TPC only & 45.4 & $\begin{array}{c}56.6 \\
{[92.6]}\end{array}$ & 34.1 & $\begin{array}{c}53.1 \\
{[90.9]}\end{array}$ \\
\hline TPC + DsECal & 32.0 & $\begin{array}{c}82.6 \\
{[97.8]}\end{array}$ & 59.0 & $\begin{array}{c}89.1 \\
{[93.8]}\end{array}$ \\
\hline $\begin{array}{l}\text { TPC + barrel } \\
\text { ECal }\end{array}$ & 22.6 & $\begin{array}{c}86.1 \\
{[91.4]}\end{array}$ & 6.9 & $\begin{array}{c}88.6 \\
{[86.5]}\end{array}$ \\
\hline
\end{tabular}

particles, the multivariate analysis quantity $R_{\mathrm{MIP} / \mathrm{EM}}$

is used. These tracks must have $R_{\mathrm{MIP} / \mathrm{EM}}>0$.

Table III shows the performance of the different PID cuts, and highlights the effectiveness of combining the TPC and $\mathrm{ECal}$ information.

The momentum distribution of the particles passing the PID cuts is shown in Fig. 8. 99.9\% of muons are rejected by the PID cuts, and the sample is $92 \%$ pure in electrons. Although a high-purity sample of electrons has been selected, $65 \%$ of the tracks arise from $\gamma \rightarrow e^{+} e^{-}$conversions in the FGD, and only $27 \%$ are from $\nu_{e}$ CC interactions. The majority of the photons come from neutrino interactions upstream of the FGD in which the conversion occurred.

To reduce the contamination from these photons, veto cuts are applied to require no reconstructed tracks in the P0D, TPC or barrel ECal in the same bunch, starting more than $100 \mathrm{~mm}$ upstream of the initial position of the electron candidate.

An additional cut in the selection removes electrons that are part of an $e^{+} e^{-}$pair. The event is rejected if there is a positive track which is electronlike $\left(\left|\delta_{e}\right|<3\right)$, starts within $100 \mathrm{~mm}$ of the electron candidate, and if the $e^{+} e^{-}$pair has an invariant mass of less than $100 \mathrm{MeV} / \mathrm{c}^{2}$. These cuts reduce the $\gamma \rightarrow e^{+} e^{-}$contamination from $65 \%$ to $30 \%$.

To further improve the $\nu_{e}$ purity, additional selections are applied and the sample is separated into CCQE-like and CCnonQE-like categories. The first mainly contains $\nu_{e}$ CCQE interactions while the latter is dominated by $\nu_{e} \mathrm{CC}$ interactions producing pions in the final state.

The CCQE-like selection requires the absence of other tracks in the TPC, except the electron candidate itself. If the electron candidate starts in FGD1 then there must be no isolated reconstructed tracks in FGD1 and no Michel electrons coming from pion decays (identified as delayed hits in FGD1). These two requirements do not apply to events in FGD2 as the lower number of scintillator layers reduces the ability to reconstruct tracks and identify delayed hits.

If the electron candidate starts in FGD2 there must be no activity in the ECal, except that caused by the electron
PHYSICAL REVIEW D 89, 092003 (2014)

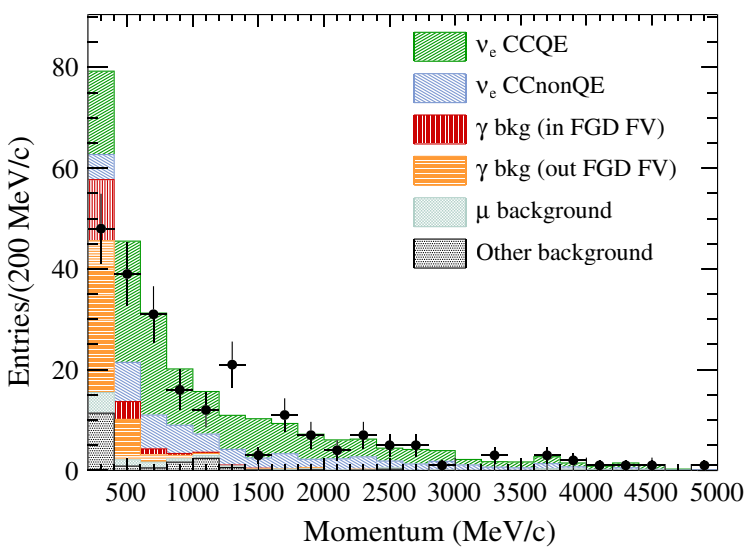

FIG. 9 (color online). Reconstructed electron momentum of events selected in the CCQE-like selection. The error on the points is the statistical error on the data, and the simulation is divided into the same categories as Fig. 8.

candidate. This cut is only applied to events in FGD2, as electrons from FGD1 can shower in FGD2 and can cause additional ECal activity not associated with the original electron candidate track.

The CCnonQE-like selection requires the presence of at least one other track which starts close to the electron candidate (within $50 \mathrm{~mm}$ ). As in the CCQE-like selection, only FGD-TPC tracks are considered for FGD2 events, whereas FGD-only tracks are also considered for FGD1 events. For FGD1 events the presence of a Michel electron in the FGD is used to tag CCnonQE-like candidates.

The final CCQE-like and CCnonQE-like selections are shown in Fig. 9 and Fig. 10 respectively. The overall efficiency of selecting $\nu_{e} \mathrm{CC}$ interactions is $26 \%$, and the efficiency of the selections as a function of $\nu_{e}$ energy is shown in Fig. 11. The purity of the selections and the predicted number of selected events are shown in Table IV.

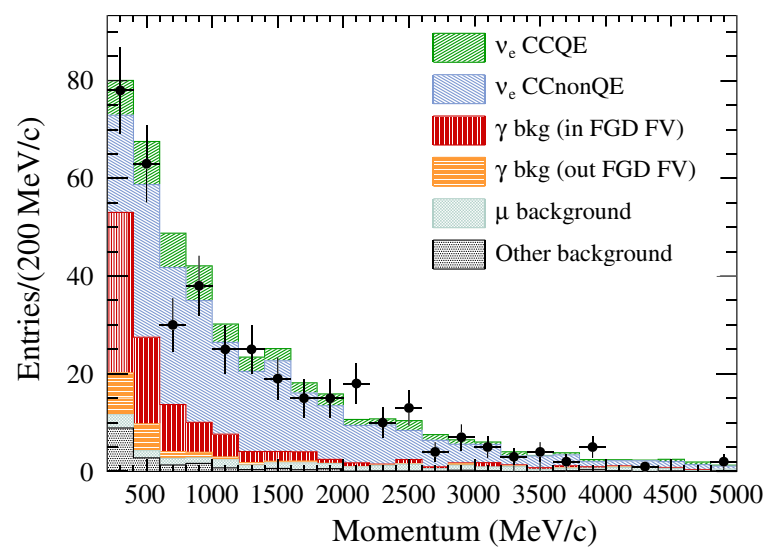

FIG. 10 (color online). Reconstructed electron momentum of events selected in the CCnonQE-like selection. The error on the points is the statistical error on the data, and the simulation is divided into the same categories as Fig. 8. 


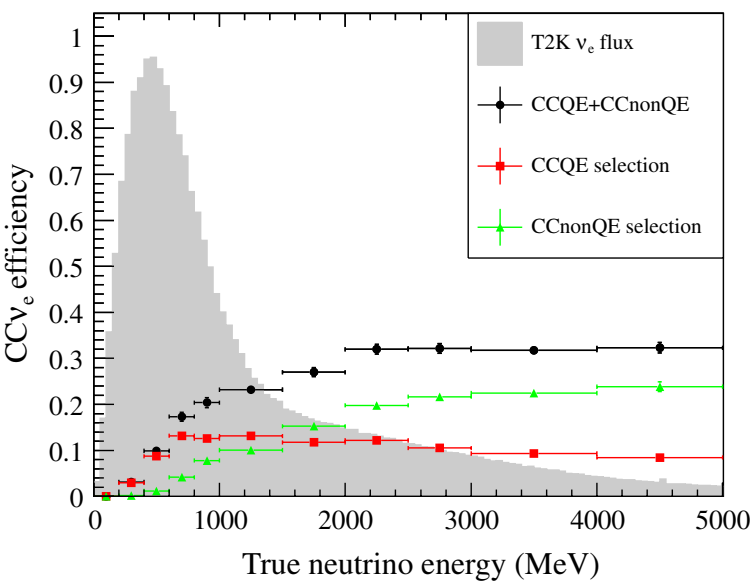

FIG. 11 (color online). Efficiency of selecting $\nu_{e} \mathrm{CC}$ interactions as a function of true neutrino energy, and the predicted $\nu_{e}$ flux at ND280.

\section{CONTROL OF THE BACKGROUNDS}

The selection of $\nu_{e} \mathrm{CC}$ interactions is designed to reject two large backgrounds. The first one is due to the muons produced in $\nu_{\mu} \mathrm{CC}$ interactions that are the dominant component of the T2K beam. This component is rejected using the PID capabilities of ND280. The second background is due to the conversions of photons in the FGD producing electrons in the TPC and it cannot be rejected using PID algorithms.

For the muon background, the combined PID of the TPC and ECal is vital to reject $99.9 \%$ of the muons. Such a large muon rejection power has been verified using a clean, datadriven sample of muons, as described in Sec. VA below. The photon background is constrained using a selection of photon conversions in the FGD in which both the electron and the positron are reconstructed in the TPC, as described in Sec. V B below.

\section{A. Muon misidentification}

A data-driven study has been carried out to confirm the muon rejection power expected by simulation. A clean sample of muons, produced by neutrino interactions in the

TABLE IV. Fractions, expected, and observed number of events for the CCQE and CCnonQE selections.

\begin{tabular}{|c|c|c|c|c|}
\hline \multirow[b]{2}{*}{ Category } & \multicolumn{2}{|c|}{ CCQE selection } & \multicolumn{2}{|c|}{ CCnonQE selection } \\
\hline & Fraction $(\%)$ & Events & Fraction $(\%)$ & Events \\
\hline$\nu_{e} \mathrm{CCQE}$ & 48.2 & 132.6 & 12.7 & 56.8 \\
\hline$\nu_{e} \mathrm{CCnonQE}$ & 19.6 & 54.1 & 52.8 & 234.7 \\
\hline$\gamma$ bkg (in FGD) & 6.4 & 17.8 & 19.2 & 85.3 \\
\hline$\gamma$ bkg (out FGD) & 15.0 & 41.4 & 4.5 & 19.9 \\
\hline$\mu$ background & 4.0 & 10.9 & 6.2 & 27.6 \\
\hline Other background & 6.8 & 18.8 & 4.6 & 20.6 \\
\hline Total simulation & 100.0 & 275.6 & 100.0 & 444.9 \\
\hline Data & & 225 & & 392 \\
\hline
\end{tabular}

sand or in the concrete walls of the ND280 pit, is selected. The selection is done by requiring one and only one track in a bunch with negative charge crossing all the 3 TPCs and starting at the upstream edge of the P0D. The TPC PID of the selected track must be compatible with a muon in the TPC upstream of the first FGD (TPC1). This requirement does not bias the sample since the TPC1 PID is not used in the analysis. Once a clean sample of muons is selected from the data, the muon misidentification probability is computed as the ratio between the number of tracks passing the PID selection and all the selected tracks. The same PID selections described in Sec. IV for the cases with and without ECal information are used. In Fig. 12 the misidentification probability as a function of the track momentum measured in the TPC is shown. The muon misidentification probability is below $1 \%$ for all the momenta and is much smaller if the TPC and the ECal PID are combined. Compatible results are obtained using simulated data confirming that the PID performances of ND280 detectors are well understood and well reproduced in the simulation.

\section{B. Photon selection}

The background, especially at low momentum, is dominated by electrons coming from photon conversions. Those electrons are background to the $\nu_{e} \mathrm{CC}$ analysis as they typically come from $\nu_{\mu}$ interactions that occur inside or outside the FGD producing a $\pi^{0}$ in the final state which immediately decays into two photons. One of the two photons then converts inside the FGD producing an $e^{+} e^{-}$ pair. If the positron is not reconstructed in the TPC the event is topologically equivalent to a $\nu_{e} \mathrm{CC}$ interaction.

This background can be estimated using a selection of photon conversions in which both the electron and the positron are reconstructed. A typical example of a photon conversion with the $e^{+} e^{-}$pair reconstructed in the TPC is shown in Fig. 13.

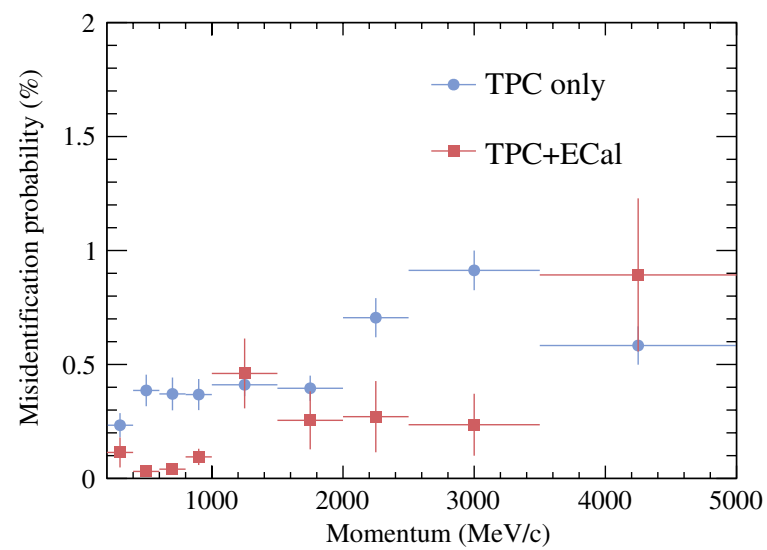

FIG. 12 (color online). Muon misidentification probability as a function of the muon momentum estimated using a sample of through going muons for the case in which the TPC PID only is used and for the case in which TPC and ECal PID are combined. 


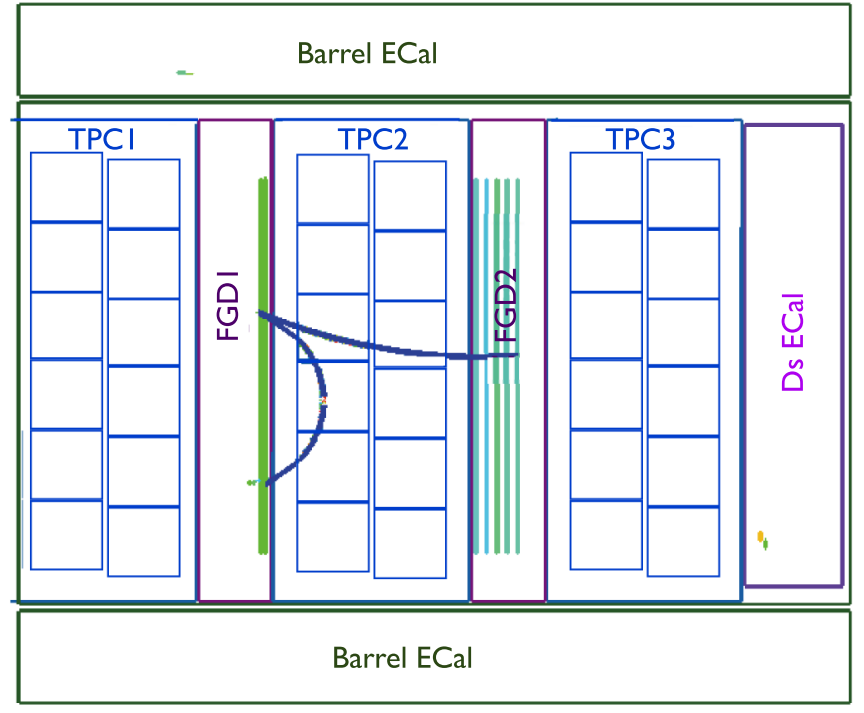

FIG. 13 (color online). Side view of a photon conversion in the FGD1 with an $e^{+} e^{-}$pair reconstructed in TPC2.

To select these events, two tracks are required to start in the FGD fiducial volume and to have opposite charge. The same data quality criteria described in Sec. IV are also required.

Both tracks have to be electronlike $\left(\left|\delta_{e}\right|<2\right.$ for the negatively charged track and $\left|\delta_{e}\right|<3$ for the positively charged track), the distance of the starting point of the two tracks is required to be within $100 \mathrm{~mm}$, and the reconstructed invariant mass of the pair has to be smaller than $50 \mathrm{MeV} / \mathrm{c}^{2}$.

The most powerful requirement among those is the invariant mass cut that alone is able to select a sample with a $90 \%$ purity in electrons. After applying all the criteria a sample with an electron purity of $99 \%$ is selected. The momentum of the electrons in the selected events is shown in Fig. 14. The purity of photon conversions in the sample is $92 \%$ with the remaining events mainly coming

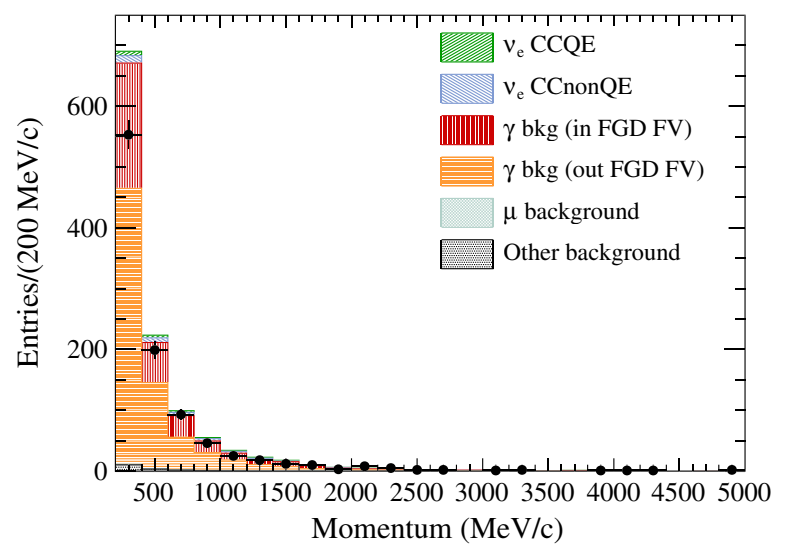

FIG. 14 (color online). Reconstructed electron momentum of events selected in the photon selection. The error on the points is the statistical error on the data, and the simulation is divided into the same categories as Fig. 8.
TABLE V. Fractions (in \%) of the different interaction types and of the production point for the events selected in the photon selection and in the photon background to the $\nu_{e} \mathrm{CC}$ selection.

\begin{tabular}{lcc}
\hline \hline Interaction & $\gamma$ background & \\
(Production point) & in $\nu_{e}$ CC selection & $\gamma$ selection \\
\hline CCQE & 4.3 & 4.1 \\
CC $1 \pi$ & 14.2 & 11.5 \\
CC coherent & 0.5 & 0.4 \\
CC other & 43.5 & 41.7 \\
NC $1 \pi^{0}$ & 8.6 & 10.9 \\
NC other & 28.8 & 31.4 \\
Inside FGD FV & 57.0 & 30.6 \\
Outside FGD FV & 43.0 & 69.4 \\
\hline \hline
\end{tabular}

from $\nu_{e}$ interactions in which the electron showers in the FGD and produces a positron in the TPC. The efficiency of this selection with respect to the total number of photons converting in the FGD is $12 \%$.

The purpose of this selection is to estimate the number of electrons coming from photon conversions entering the $\nu_{e}$ $\mathrm{CC}$ selection. In order to do this it is necessary to ensure that the characteristics of the events selected in the photon selection and in the photon background in the $\nu_{e} \mathrm{CC}$ selection are similar. Specifically they need to have the same origin, they need to be produced in the same type of neutrino interactions, and the selected lepton in the two cases needs to cover the same phase space. Table V shows the neutrino interactions contributing to the two samples and the fraction of neutrinos interacting inside or outside the FGD. The fractions of neutrino interactions are similar while the photon selection has more events coming from outside of the FGD. This difference is due to the different geometrical acceptance of the two samples because the photon selection requires both tracks to enter the TPC while the $\nu_{e} \mathrm{CC}$ selection requires only one TPC track.

The lepton momentum and angle, and the neutrino energy are in reasonable agreement between the two selections. The strategy used to constrain the background in the extraction of the beam $\nu_{e}$ component will be described in Sec. VII.

\section{SYSTEMATIC UNCERTAINTIES}

The systematic uncertainties in the measurement of the beam $\nu_{e}$ component are separated into three main categories: detector performance, external backgrounds, and neutrino flux and cross section uncertainties. The systematic uncertainties described in this section are used as prior constraints in the fit to extract the beam $\nu_{e}$ component that will be described in Sec. VII.

\section{A. Detector systematic uncertainties}

The detector systematic uncertainties are computed for each subdetector used in this analysis: the TPCs, the FGDs 
and the different ECal modules. Systematic effects related to neutrino interactions outside ND280 producing particles entering the detector are also considered in this class of systematics.

To determine the effect of the detector systematic uncertainties on the analysis 1000 toy experiments have been performed. Each toy experiment has a set of detector systematic parameter values drawn from Gaussian distributions. The 1000 toy experiments are used to evaluate the covariance of the number of events in bins of reconstructed electron momentum and neutrino flavor for each selection. Seven bins are included for the CCQE-like and CCnonQElike selection and 3 bins for the $\gamma$ selection.

\section{TPC systematic uncertainties}

TPC systematic uncertainties are divided into three classes: selection efficiency, momentum resolution, and particle identification.

The efficiency systematic uncertainty arises from the cluster finding, track finding and charge assignment. It is evaluated using events with a single particle passing through multiple TPCs, to check for the presence of a reconstructed track with the correct charge assignment. The TPC reconstruction efficiency is determined to be $\left(99.8_{-0.4}^{+0.2}\right) \%$ and the charge misassignment probability is below $1 \%$ for tracks with momenta less than $5 \mathrm{GeV} / \mathrm{c}$.

Momentum reconstruction is affected by nonuniformity of the magnetic field and the overall magnetic field strength. The field inside the magnet has been measured with a Hall probe and nonuniformities are checked with photoelectrons produced by shining a laser at the central cathode of the TPC that has small aluminium dots on it. Uncertainty in the overall magnetic field strength leads to an uncertainty on the momentum scale of $0.6 \%$. An additional source of systematic uncertainty is the momentum resolution that has been determined using tracks crossing multiple TPCs and comparing their reconstructed momenta. The inverse momentum resolution is found to be significantly better in simulations than in data, which could be due to nonuniformity of the electric field. These nonuniformities depend on the drift distance and cancel out for tracks close to the cathode. For this reason they are not observed in the analysis of photoelectrons produced by the laser. At momenta larger than $1.4 \mathrm{GeV} / \mathrm{c}$, the inverse momentum resolution is $(30 \pm 10) \%$ larger in data than in simulation.

Systematic uncertainties on the TPC particle identification are computed using high-purity samples of electrons, muons and protons. By definition the electron sample should give a $\delta_{e}$ distribution that is Gaussian with mean 0 and width 1 . The simulation and the data have a difference on the mean of the pull distribution of $(-0.12 \pm 0.12)$, and a scale of the width of $(1.02 \pm 0.07)$. These are converted into systematic uncertainties on the energy loss for each true electron track, and all the TPC PID pulls are recomputed. Similarly, the $\delta_{p}$ distribution of a proton sample is used to determine the energy loss systematic uncertainties of true protons. The $\delta_{\mu}$ distribution of the through-going muon sample is used for both muons and pions, as their masses are similar.

\section{FGD systematic uncertainties}

The systematic uncertainties related to the FGDs arise from potential mismodeling of the track-finding efficiency, the efficiency with which TPC and FGD tracks are matched, the Michel electron tagging efficiency, secondary pion interactions, and the FGD mass.

The efficiency with which FGD tracks are reconstructed is computed for FGD1, as the analysis uses isolated FGD reconstruction for FGD1 only. To determine the efficiency, samples of stopping protonlike tracks in FGD1 are used and differences between the data and the simulation are evaluated for different angles, being 3\% for forward-going tracks and rising to $21 \%$ for high angle tracks. Additional studies have been done for multiple tracks in the FGD by using hybrid samples in which simulated particles (pions or protons) are injected into data and simulated events finding differences between data and simulation of 3\% (4\%) for pions (protons).

The TPC-FGD matching efficiency is studied using a sample of through-going particles, in which the presence of a track in the TPC upstream and downstream implies that a track should be seen in the FGD. The efficiency is found to be $99.9 \%$. The matching efficiency has also been checked for electrons using samples of photon conversions in the FGD in which the $e^{+} e^{-}$tracks were not required to start in the FGD.

The Michel electron tagging efficiency is studied using a sample of cosmic rays which stop in FGD1. The particles must have a range compatible with being a muon with the momentum as reconstructed in the TPC. The Michel tagging efficiency is found to be about $60 \%$, depending on the beam bunch of the primary neutrino interaction.

There is an uncertainty in the modeling of pion reinteractions (where a pion created in an interaction is ejected from the nucleus and interacts with another nucleus in the detector). Differences between external pion interaction data [40] and GEANT4 simulation are evaluated. The external data are extrapolated to cover the whole momentum region relevant for $\mathrm{T} 2 \mathrm{~K}$ and a correction weight is calculated for each event, along with an uncertainty based on the error of the extrapolated data. This systematic uncertainty can only migrate events between the CCQElike and the CCnonQE-like selections and the effect on this analysis is found to be negligible.

The uncertainty on the mass of the FGDs-and thus on the number of target nucleons-is computed using the known density and size of the individual components, and their uncertainties. The overall uncertainty on the FGD mass is $0.67 \%$. 


\section{ECal systematic uncertainties}

ECal systematic uncertainties are computed for the particle identification, the energy resolution and scale, and the efficiency with which ECal objects are reconstructed and matched to TPC tracks.

Uncertainties in $R_{\mathrm{MIP} / \mathrm{EM}}$ are calculated using high-purity samples of electrons and muons. The efficiency of the $R_{\text {MIP/EM }}>0$ requirement is calculated for data and for the simulation, and the difference is taken as the systematic uncertainty. The systematic uncertainty for selecting electrons is $2.1 \%$ in the DsECal and $2.9 \%$ in the barrel, and the uncertainty for rejecting muons is $0.6 \%$ in the DsECal and $0.7 \%$ in the barrel ECal.

$E_{\mathrm{EM}}$ systematic uncertainties are computed using both a high-purity sample of electrons from $\mathrm{T} 2 \mathrm{~K}$ data and test beam data taken at CERN before the DsECal was shipped to Japan. The reconstructed $E_{\mathrm{EM}}$ is compared to the known momentum, either as measured by the TPC for the in situ sample, or as defined in the test beam. The uncertainty on the energy scale is $6 \%$ and the uncertainty on the energy resolution is $15 \%$.

A combined systematic uncertainty is computed for the efficiency of reconstructing an ECal object and matching it to a TPC track. A high-purity sample of electrons that appear to enter the ECal (by extrapolating the TPC track) is used. The uncertainty is $1.6 \%$ for tracks entering the DsECal, and $3.4 \%$ for tracks entering the barrel ECal.

\section{B. External background systematic uncertainties}

\section{Systematic uncertainties from neutrino interactions outside the FGD FV}

In the $\nu_{e} \mathrm{CC}$ selection there is a large background component coming from $\nu_{\mu}$ interactions outside of the FGD. Differences between data and simulation in this component might arise from mismodeling of the $\pi^{0}$ production and in the description of the material and efficiencies outside of the FGD. The fit to the ND280 $\nu_{\mu}$ CC samples cannot constrain this component as $<5 \%$ of the events selected in that analysis come from outside of the FGD.

In this analysis, the majority of the selected events arising from neutrino interactions outside of the FGD are due to photon conversion in the FGD and this component is well measured by the photon selection. The remainder of this background is due to other charged particles (pions or protons) and this component cannot be measured by the photon selection. The systematic uncertainty has been evaluated with control samples in which different sources of external backgrounds producing tracks reconstructed as starting inside the FGD were selected. The systematic uncertainty is set to $30 \%$ for each of the two components.

\section{Systematic uncertainties from neutrino interactions outside ND280}

The standard ND280 simulation only includes neutrino interactions inside the magnet volume. Neutrino interactions outside this volume can affect the analysis in two different ways. First, the particles produced in these interactions can enter the ND280 volume, interact in an FGD, and be selected as signal. A separate simulation of neutrino interactions outside the ND280 magnet is used to estimate the expected number of extra signal events and is found to be negligible. Particles produced outside ND280 can also enter in one of the ND280 subdetectors in the same beam bunch in which a true $\nu_{e}$ signal interaction occurs in an FGD. These particles can then cause an event to be vetoed, reducing the selection efficiency in data. The probability of this occurring is calculated separately for each veto cut and for each $\mathrm{T} 2 \mathrm{~K}$ run, as it depends on the beam intensity. The largest correction is $(2.3 \pm 0.5) \%$ for the ECal veto cut in run IV.

\section{Flux and cross section systematic uncertainties}

For the $\mathrm{T} 2 \mathrm{~K}$ oscillation analyses, the flux and the cross section systematic uncertainties described in Sec. II are evaluated using a selection of $\nu_{\mu} \mathrm{CC}$ interactions in ND280 [3]. Events with a muon candidate produced in FGD1 and entering the downstream TPC are divided into the following samples:

(i) $\mathrm{CC} 0 \pi$ sample in which there are no tracks compatible with a pion. Most of the events arise from CCQE interactions;

(ii) $\mathrm{CC} 1 \pi$ sample in which there is one reconstructed track (in the TPC or in the FGD) compatible with a pion or there is a delayed energy deposit consistent with a stopping pion. It is mainly composed of CC interactions with resonant pion production;

(iii) CC-other sample, for all other topologies. Most events are from deep inelastic scattering and multipion production.

These three samples are fit to evaluate the $\nu_{\mu}$ flux and cross section parameters and their uncertainties. The oscillation analyses also use these samples to evaluate the $\nu_{e}$ flux parameters, which is possible because $\nu_{\mu}$ and $\nu_{e}$ arise from the same parent particles. The cross sections for $\nu_{e}$ and $\nu_{\mu}$ interactions are assumed to be the same.

The muon momentum distributions for the $\mathrm{CCO} \pi$ and $\mathrm{CC} 1 \pi$ samples are shown in Fig. 15 together with the prediction before and after the fit.

The systematic parameters and their uncertainties obtained from the fit to the ND280 $\nu_{\mu}$ CC data are shown in Table VI. The effect of those systematic uncertainties on the evaluation of the beam $\nu_{e}$ component is $6 \%$, as will be shown in Sec. VIII. Without using the ND280 $\nu_{\mu}$ data the uncertainty on the beam $\nu_{e}$ prediction is larger than $20 \%$.

In the analysis described in this paper, systematic uncertainties of final state interactions are not evaluated 
MEASUREMENT OF THE INTRINSIC ELECTRON ...
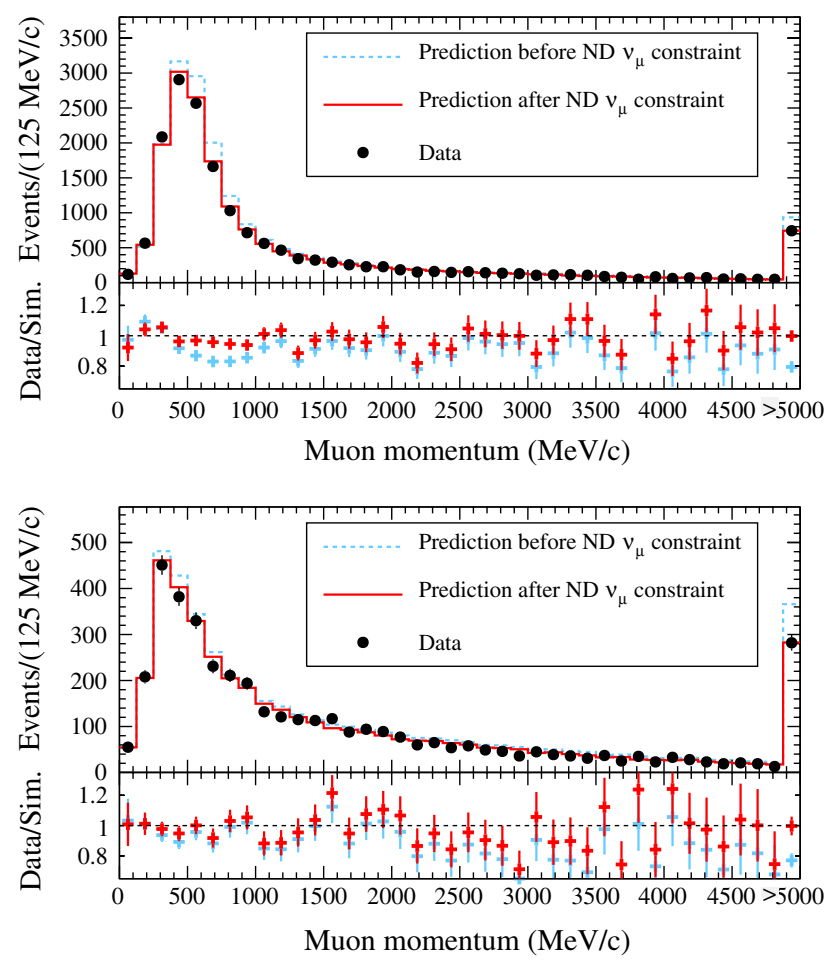

FIG. 15 (color online). Reconstructed momentum distribution for events selected in the ND280 $\nu_{\mu} \mathrm{CC}$ analysis in the CC0 $\pi$ sample (top) and in the $\mathrm{CC} 1 \pi$ sample (bottom) before and after the fit to evaluate flux and cross section parameters. The last bins contain all the events with reconstructed muon momentum larger than $5 \mathrm{GeV} / \mathrm{c}$. The error on the points is the statistical error on the data.

from the ND280 $\nu_{\mu} \mathrm{CC}$ samples; their effect is evaluated in bins of reconstructed electron momentum using the pion scattering data described in Sec. II B.

\section{Summary of the systematic uncertainties}

The 60 parameters included in this analysis are shown in Table VI. The variance due to limited statistics in the simulation is added to the diagonal of the detector and FSI parameter covariance matrix.

\section{MEASUREMENT OF THE ELECTRON NEUTRINO COMPONENT OF THE BEAM}

In order to extract the beam $\nu_{e}$ component, a likelihood fit of the selected distributions (CCQE-like, CCnonQElike and $\gamma$ samples) has been performed. To account for systematic uncertainties, 60 nuisance parameters are included in the likelihood function. An additional term, $R\left(\nu_{e}\right)$, is included that scales the expected number of events arising from $\nu_{e}$ interactions. A second approach introduces two additional terms, $R\left(\nu_{e}(\mu)\right)$ and $R\left(\nu_{e}(K)\right)$, which separately scale the expected number of $\nu_{e}$ events arising from muon and kaon decays respectively.

It is important to notice that in this analysis the cross section parameters shown in Table VI have been evaluated
PHYSICAL REVIEW D 89, 092003 (2014)

TABLE VI. Summary of the values and errors of the systematic parameters used as priors for the measurement described in this paper. The values in squared brackets show the range of variation for systematic uncertainties with more than one parameter as a function of neutrino energy.

\begin{tabular}{lccc}
\hline \hline Systematic & $\begin{array}{c}\text { Central } \\
\text { value }\end{array}$ & Uncertainty & $\begin{array}{c}\text { Number of } \\
\text { parameters }\end{array}$ \\
\hline$\nu_{\mu}$-flux & {$[0.93-1.05]$} & {$[0.07-0.08]$} & 11 \\
$\nu_{e}$-flux & {$[0.95-1.02]$} & {$[0.07-0.09]$} & 7 \\
$\bar{\nu}_{\mu}$-flux & {$[0.99-1.03]$} & {$[0.09-0.14]$} & 5 \\
$\bar{\nu}_{e}$-flux & {$[0.95-1.01]$} & {$[0.08-0.17]$} & 2 \\
$M_{A}^{\mathrm{QE}}[\mathrm{GeV}]$ & 1.24 & 0.07 & 1 \\
$x_{1,2,3}^{\mathrm{QE}}$ & {$[0.85-0.97]$} & {$[0.07-0.11]$} & 3 \\
$x_{\mathrm{SF}}$ & 0.24 & 0.13 & 1 \\
$p_{F}[\mathrm{MeV} / \mathrm{c}]$ & 266 & 11 & 1 \\
$E_{b}[\mathrm{MeV}]$ & 30.9 & 5.2 & 1 \\
$M_{A}^{\mathrm{RES}}[\mathrm{GeV}]$ & 0.96 & 0.07 & 1 \\
$x_{1,2}^{\mathrm{CC} 1 \pi}$ & {$[1.12-1.26]$} & {$[0.16-0.17]$} & 2 \\
$x^{\mathrm{NC} 1 \pi^{0}}$ & 1.14 & 0.25 & 1 \\
$x^{\mathrm{NC} \text { other }}$ & 1.41 & 0.22 & 1 \\
$x^{\pi \text {-less }}$ & 0.21 & 0.09 & 1 \\
$x^{\mathrm{CC} \text { coh }}$ & 0.45 & 0.16 & 1 \\
$x^{\mathrm{CC} \text { other }}(\mathrm{GeV})$ & 0.23 & 0.29 & 1 \\
$\sigma_{\bar{\nu}} / \sigma_{\nu}$ & 1 & 0.4 & 1 \\
Detector $+\mathrm{FSI}$ & 1 & {$[0.07-0.19]$} & 17 \\
Out FGD FV $e^{-}$ & 1 & 0.3 & 1 \\
Out FGD FV other & 1 & 0.3 & 1 \\
Total & & & 60 \\
\hline \hline
\end{tabular}

from the ND280 $\nu_{\mu} \mathrm{CC}$ samples assuming that the effective cross section parameters for $\nu_{\mu}$ and $\nu_{e}$ interactions are the same. A measurement of the $\nu_{e} \mathrm{CC}$ interactions at ND280 is important to put experimental constraints on this assumption that is poorly constrained from experimental data [11].

The likelihood ratio applied to the momentum distributions of the $\nu_{e}$ CCQE-like, $\nu_{e}$ CCnonQE-like and $\gamma$ samples is

$$
\begin{aligned}
-2 \ln \lambda(R ; \vec{f})= & -2 \ln \lambda_{\nu_{e} \mathrm{CCQE}}(R ; \vec{f})-2 \ln \lambda_{\nu_{e} \mathrm{CCnonQE}}(R ; \vec{f}) \\
& -2 \ln \lambda_{\gamma}(R ; \vec{f})+\left(\vec{f}-\vec{f}_{0}\right)^{T} V^{-1}\left(\vec{f}-\vec{f}_{0}\right)
\end{aligned}
$$

where $-2 \ln \lambda_{i}$ is the likelihood ratio for each sample and the last term is the penalty term that constrains the systematic nuisance parameters. $\vec{f}_{0}$ is the vector of central values for the systematic parameters shown in Table VI, $\vec{f}$ is the vector of nuisance parameters and $V$ is the covariance matrix that takes into account the correlations among the systematics parameters.

The likelihood function is calculated in 18 bins of reconstructed electron momentum from $200 \mathrm{MeV} / \mathrm{c}$ to 
$10 \mathrm{GeV} / \mathrm{c}$ for each selection, with a $100 \mathrm{MeV} / \mathrm{c}$ bin width up to $1 \mathrm{GeV} / \mathrm{c}$ and a wider binning at higher momenta.

The predicted number of events in each bin depends on the free parameter $R\left(\nu_{e}\right)$ and on the 60 systematic parameters according to the formula:

$n_{\text {exp }}^{i}(R ; \vec{f})=\frac{N_{\text {p.o.t. }}^{\text {data }}}{N_{\text {p.o.t. }}^{\text {sim }}} \cdot \sum_{j}^{N_{i}} f_{\text {flux }}^{j} f_{x(\text { norm })}^{j} f_{x(\text { resp })}^{j} f_{\text {det }}^{j}\left(p_{i}\right) \times R\left(\nu_{e}\right)$.

The sum $j$ runs over the events with momentum compatible with the $i$ th momentum bin $p_{i}$, with $N_{i}$ total events in that bin. $N_{\text {p.o.t. }}^{\text {data }}$ and $N_{\text {p.o.t. }}^{\text {sim }}$ are the number of p.o.t. for data and simulation. $f_{\text {flux }}$ multiply the flux prediction in bins of true neutrino energy. $f_{\text {det }}$ multiply the expected number of events in bins of electron reconstructed momentum. The cross section parameters are treated in two ways: $f_{x(\text { norm })}$ multiply the cross section normalization for a given true neutrino energy and interaction model while $f_{x(\text { resp })}$ are precalculated response functions whose value for the nominal parameter settings of Table VI is one. The reason for which they are treated in this way is that they can have a nonlinear dependency on the cross section parameters. The prediction for the number of selected events before and after the application of the detector systematic and of the flux and cross section parameters obtained from the fit to the ND280 $\nu_{\mu}$ CC samples is shown in Table VII.

For each of the three samples $k$ the likelihood ratio is

$$
\begin{aligned}
-2 \ln \lambda_{k}(R ; \vec{f})= & 2 \times \sum_{i=1}^{18}\left\{n_{\text {exp }}^{i}(R ; \vec{f})-n_{\text {data }}^{i}\right. \\
& \left.+n_{\text {data }}^{i} \ln \left(n_{\text {data }}^{i} / n_{\exp }^{i}(R ; \vec{f})\right)\right\}
\end{aligned}
$$

where $n_{\exp }^{i}$ and $n_{\text {data }}^{i}$ are the number of expected and observed events in the $i$ th momentum bin.

TABLE VII. Number of events for CCQE-like and CCnonQElike selections before and after the fit to the ND280 $\nu_{\mu} \mathrm{CC}$

\begin{tabular}{|c|c|c|c|c|}
\hline \multirow[b]{2}{*}{ Category } & \multicolumn{2}{|c|}{ CCQE selection } & \multicolumn{2}{|c|}{ CCnonQE selection } \\
\hline & Before & After & Before & After \\
\hline$\nu_{e}$ from $\mu$ & 61.2 & 52.7 & 38.9 & 34.9 \\
\hline$\nu_{e}$ from $\mathrm{K}$ & 125.9 & 108.2 & 253.6 & 221.0 \\
\hline$\nu_{\mu}($ in $\mathrm{FV})$ & 30.4 & 31.7 & 124.9 & 132.4 \\
\hline$\nu_{\mu}(\mathrm{OOFV})$ & 58.1 & 67.0 & 27.5 & 26.8 \\
\hline Total & 275.5 & 259.6 & 444.9 & 415.1 \\
\hline Data & \multicolumn{2}{|c|}{225} & \multicolumn{2}{|c|}{392} \\
\hline
\end{tabular}
samples.

\section{RESULTS}

The result obtained for the parameter $R\left(\nu_{e}\right)$ is

$$
\begin{aligned}
R\left(\nu_{e}\right)= & 1.01 \pm 0.06(\text { stat }) \pm 0.06(\text { flux } \oplus x . \text { sec }) \\
& \pm 0.05(\operatorname{det} \oplus \text { FSI }) \\
= & 1.01 \pm 0.10
\end{aligned}
$$

where the first term represents the statistical error, the second term represents the systematic uncertainties related to the flux and to the cross section models, and the last term represents the detector systematics and the FSI uncertainties.

This result indicates that the beam $\nu_{e}$ component measured in the data is consistent with the expectation for this component after the constraint from the ND280 $\nu_{\mu}$ $\mathrm{CC}$ sample. This is a key validation of the strategy followed by $\mathrm{T} 2 \mathrm{~K}$ to constrain the flux and cross section parameters for all the neutrino oscillation analyses.

The second measurement is performed by fitting independently the $\nu_{e}$ originating from muons and from kaons. As is shown in Fig. 1 the $\nu_{e}$ from muons mainly populate the low energy region while the $\nu_{e}$ from kaons are dominant at high momenta.

Given the larger efficiency of the analysis at high energy (shown in Fig. 11) there are three times as many selected events from kaon decay as from muon decay. The $\nu_{e}$ from muon decay mainly populate the CCQE-like selection in its low momentum region. The results obtained for the two components are

$$
\begin{aligned}
R\left(\nu_{e}(\mu)\right)= & 0.68 \pm 0.24(\text { stat }) \pm 0.11(\text { flux } \oplus x . \text { sec }) \\
& \pm 0.14(\operatorname{det} \oplus \text { FSI }) \\
= & 0.68 \pm 0.30
\end{aligned}
$$

and

$$
\begin{aligned}
R\left(\nu_{e}(K)\right)= & 1.10 \pm 0.08(\text { stat }) \pm 0.09(\text { flux } \oplus x . \mathrm{sec}) \\
& \pm 0.06(\operatorname{det} \oplus \mathrm{FSI}) \\
= & 1.10 \pm 0.14
\end{aligned}
$$

Due to the small amount of $\nu_{e}$ coming from muons, the uncertainty on the measurement of this component is still statistically limited and will be improved when more data is available. With the present statistics both numbers are compatible with unity showing no discrepancies between the predicted and the observed beam $\nu_{e}$ component. The larger systematic uncertainty for $R\left(\nu_{e}(\mu)\right)$ is due to the fact that the detector, flux and cross section systematic uncertainties are larger at low momenta. The distribution of the reconstructed electron momentum for the three samples after the fit are shown in Fig. 16. 

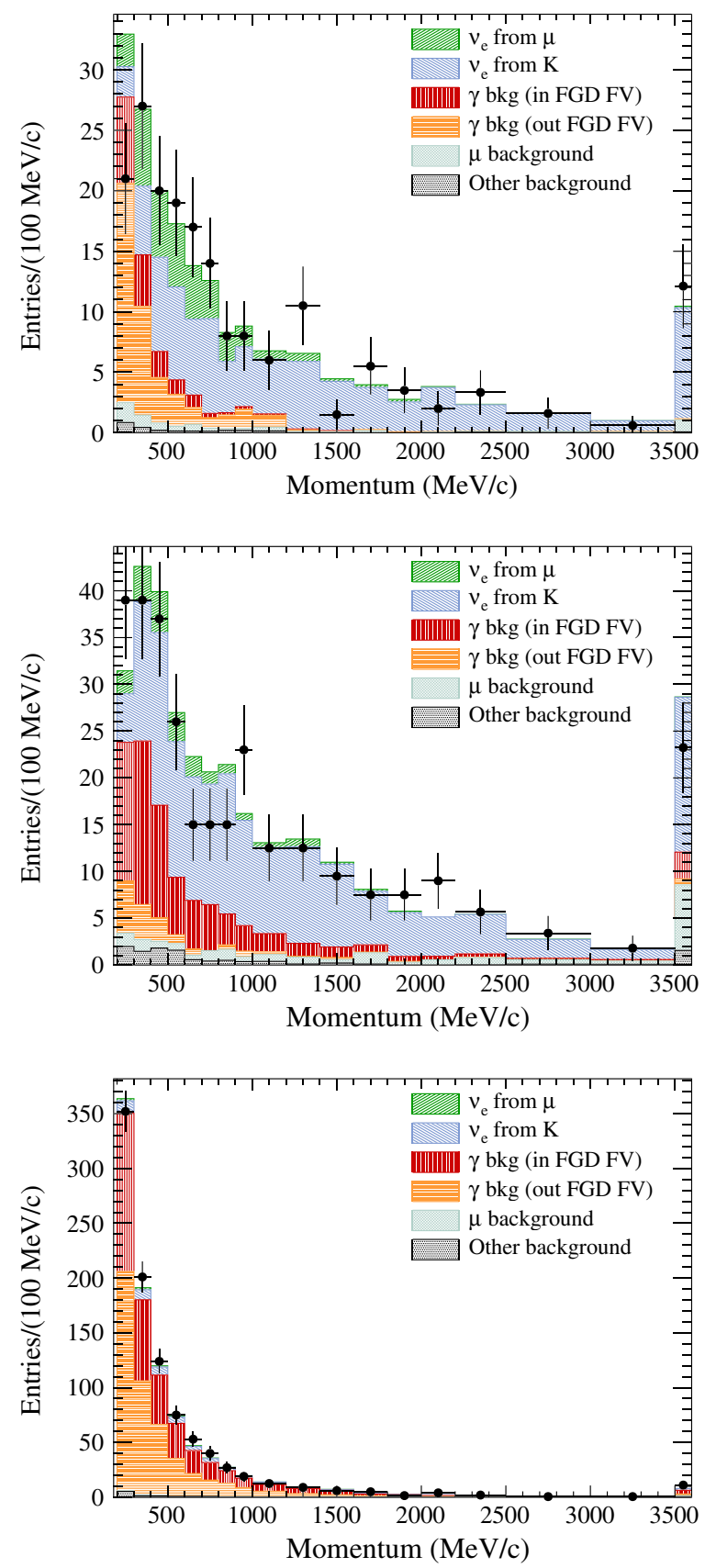

FIG. 16 (color online). Reconstructed electron momentum distribution for the events selected in the three samples after the fit to extract the beam $\nu_{e}$ component: CCQE-like selection (top), CCnonQE-like selection (center) and $\gamma$ selection (bottom). The last bin contains all the events with reconstructed electron momentum larger than $3.5 \mathrm{GeV} / \mathrm{c}$. The signal is divided into $\nu_{e}$ produced by muon and kaon decays. The background is divided into the same categories as Fig. 8. The error on the points is the statistical error on the data.

As far as the nuisance parameters are concerned, the fitted values are in good agreement with the expectations. The out of FGD electron component is reduced in the fit by $0.64 \pm 0.10$, compatible with the prior systematic uncertainty of $30 \%$. This reduction might point to the fact that the simulation does not properly reproduce the amount of $\pi^{0}$ produced in neutrino interactions in the materials surrounding the ND280 tracker region. Those interactions are mainly high energy deep inelastic scattering events for which the $\pi^{0}$ multiplicity is not well measured. This reduction does not have a large impact on the measurements presented here because of the presence of the photon conversion sample used to evaluate this background.

\section{SUMMARY}

In summary, a selection of $\nu_{e} \mathrm{CC}$ interactions has been performed using the T2K off-axis near detector combining the PID capabilities of the TPC and ECal. The combination of these two detectors allows the selection of a clean sample of electrons with a purity of $92 \%$ and a muon misidentification probability smaller than $1 \%$.

The selected sample is mainly composed of electrons coming from $\nu_{e} \mathrm{CC}$ interactions but a non-negligible component comes from photon conversions in the FGD. This background is constrained in the analysis using a sample of $e^{+} e^{-}$pairs coming from photon conversions in which both outgoing particles are reconstructed in the TPC.

To extract the beam $\nu_{e}$ component from the data a likelihood fit is performed. The expected number of $\nu_{e}$ interactions is predicted by the same model used for the T2K oscillation analyses where the neutrino fluxes and the neutrino cross sections are evaluated by the $\nu_{\mu} \mathrm{CC}$ samples selected at ND280.

The observed number of events is in good agreement with the prediction, providing a direct confirmation of this method. This measurement is still statistically limited but when additional data is collected it will be possible to further improve the measurement of the intrinsic $\nu_{e}$ component in the $\mathrm{T} 2 \mathrm{~K}$ beam and perform measurements of $\nu_{e}$ cross sections and of the $\nu_{e} / \nu_{\mu}$ cross section differences that have not been measured at T2K energies.

This measurement is particularly important because the intrinsic $\nu_{e}$ component is the main background for all the proposed long-baseline neutrino oscillation experiments aiming to measure $C P$ violation in the leptonic sector. In this paper it is shown that, although the component is small, it is possible to measure it with a properly designed near detector.

\section{ACKNOWLEDGMENTS}

We thank the J-PARC staff for superb accelerator performance and the CERN NA61 Collaboration for providing valuable particle production data. We acknowledge the support of MEXT, Japan; NSERC, NRC and CFI, Canada; CEA and CNRS/IN2P3, France; DFG, Germany; INFN, Italy; National Science Centre (NCN), Poland; RAS, RFBR and MES, Russia; MICINN and CPAN, Spain; SNSF and SER, Switzerland; STFC, UK; and DOE, USA. 
We also thank CERN for the UA1/NOMAD magnet, DESY for the HERA-B magnet mover system, NII for SINET4, the WestGrid and SciNet consortia in Compute Canada, and GridPP, UK. In addition participation of individual researchers and institutions has been further supported by funds from ERC (FP7), EU; JSPS, Japan; Royal Society, UK; and DOE Early Career program, USA.
[1] K. Abe et al. (T2K Collaboration), Nucl. Instrum. Methods Phys. Res., Sect. A 659, 106 (2011).

[2] J. Arafune, M. Koike, and J. Sato, Phys. Rev. D 56, 3093 (1997).

[3] K. Abe et al. (T2K Collaboration), Phys. Rev. Lett. 112, 061802 (2014).

[4] K. Abe et al. (T2K Collaboration), Phys. Rev. D 87, 012001 (2013).

[5] K. Abe et al., arXiv:1109.3262.

[6] C. Adams et al., arXiv:1307.7335.

[7] A. Stahl et al., Report No. CERN-SPSC-2012-021; SPSC-EOI-007, 2012.

[8] P. A. Amaudruz et al., Nucl. Instrum. Methods Phys. Res., Sect. A 696, 1 (2012).

[9] N. Abgrall et al., Nucl. Instrum. Methods Phys. Res., Sect. A 637, 25 (2011).

[10] D. Allan et al., JINST 8, P10019 (2013).

[11] M. Day and K. S. McFarland, Phys. Rev. D 86, 053003 (2012).

[12] D. Beavis, A. Carroll, I. Chiang et al., Physics Design Report No.52459, BNL, 1995.

[13] M. Otani et al., Nucl. Instrum. Methods Phys. Res., Sect. A 623, 368 (2010).

[14] K. Abe et al. (T2K Collaboration), Phys. Rev. Lett. 107, 041801 (2011).

[15] K. Abe et al. (T2K Collaboration), Phys. Rev. D 88, 032002 (2013).

[16] A. Ferrari, P. R. Sala, A. Fasso, and J. Ranft, FLUKA: A multi-particle transport code, Report No. CERN-2005-10 (2005), No. INFN/TC_05/11, No. SLAC-R-773.

[17] N. Abgrall et al. (NA61/SHINE collaboration), arXiv: 1401.4699.

[18] N. Abgrall et al. (NA61/SHINE collaboration), Phys. Rev. C 84, 034604 (2011).

[19] N. Abgrall et al. (NA61/SHINE collaboration), Phys. Rev. C 85, 035210 (2012).

[20] T. Eichten et al., Nucl. Phys. B44, 333 (1972).
[21] J. V. Allaby et al., Technical Report No. 70-12, CERN, 1970.

[22] R. Brun, F. Carminati, and S. Giani, Report No. CERNW5013, 1994.

[23] C. Zeitnitz and T. A. Gabriel, in Proceedings of the International Conference on Calorimetry in High Energy Physics, 1993 (unpublished).

[24] Y. Hayato, Nucl. Phys. B, Proc. Suppl. 112, 171 (2002).

[25] K. Abe et al. (T2K Collaboration), Phys. Rev. D 87, 092003 (2013).

[26] C. H. Llewellyn Smith, Phys. Rep. C3, 261 (1972).

[27] R. A. Smith and E. J. Moniz, Nucl. Phys. B43, 605 (1972).

[28] D. Rein and L. M Sehgal, Ann. Phys. (N.Y.) 133, 79 (1981).

[29] M. Gluck, E. Reya, and A. Vogt, Eur. Phys. J. C 5, 461 (1998).

[30] T. Sjostrand, Comput. Phys. Commun. 82, 74 (1994).

[31] A. Bodek and U. K. Yang, arXiv:hep-ex/0308007.

[32] A. A. Aguilar-Arevalo et al. (MiniBooNE collaboration), Phys. Rev. D 81, 092005 (2010).

[33] V. Lyubushkin et al. (NOMAD collaboration), Eur. Phys. J. C 63, 355 (2009).

[34] A. A. Aguilar-Arevalo et al. (MiniBooNE collaboration), Phys. Rev. D 83, 052007 (2011).

[35] A. A. Aguilar-Arevalo et al. (MiniBooNE collaboration), Phys. Rev. D 83, 052009 (2011).

[36] A. A. Aguilar-Arevalo et al. (MiniBooNE collaboration), Phys. Rev. D 81, 013005 (2010).

[37] M. Hasegawa et al. (K2K collaboration), Phys. Rev. Lett. 95, 252301 (2005).

[38] K. Hiraide et al. (SciBooNE collaboration), Phys. Rev. D 78, 112004 (2008).

[39] T. Golan, J. T. Sobczyk, and J. Zmuda, Nucl. Phys. B, Proc. Suppl. 229-232, 499 (2012).

[40] P. de Perio, AIP Conf. Proc. 1405, 223 (2011).

[41] S. Aoki et al., Nucl. Instrum. Methods Phys. Res., Sect. A 698, 135 (2013).

[42] S. Assylbekov et al., Nucl. Instrum. Methods Phys. Res., Sect. A 686, 48 (2012). 